\title{
Strained Premixed Laminar Flames with Nonunity Lewis Numbers
}

\author{
PAUL A. LIBBY AMABLE LIÑAN FORMAN A. WILLIAMS
}

\begin{abstract}
The method of activation energy asymptotics is used to study the effects of Lewis numbers different from unity on nonadiabatic flamelets in counterflowing streams of reactants and products. A sequence of analyses parallels those reported earlier for such flamelets having Lewis number unity. Thus initial results relate to nearly adiabatic flows with Lewis numbers close to unity. It is found that the effect of nonunity Lewis numbers is accentuated in flamelets subjected to low rates of strain and that Lewis numbers greater than unity tend to promote extinction. Thus abrupt extinction and ignition events can occur even under adiabatic conditions. Next fully nonadiabatic flamelets with Lewis numbers near unity are treated in order to consider cases involving relatively large degrees of product heating and cooling. These results relate to reaction zones as they arise under conditions of low-to-moderate rates of strain with the customary diffusivereactive balance. We also treat flamelets subjected to such high rates of strain that the reaction zone is extended and located far into the product stream. In this case a diffusive-convectivereactive balance prevails. Realistic density variations are considered in the numerical examples and are shown to tend to retard extinction.
\end{abstract}

\section{INTRODUCTION}

Previous analyses (Libby and Williams, 1982 and 1983) $\dagger$ treat laminar flamelets in variable density, counterflowing streams of reactants and products in gases with Lewis number unity by the method of activation energy asymptotics. Motivations for these studies are discussed fully in LWI and LWII; briefly, such laminar flamelets are of interest in laboratory experiments designed to provide information on overall chemical kinetic rates and in analyzing the effect of the rate of strain on the characteristics of turbulent reaction zones. In real flames the Lewis numbers are not precisely unity and under the conditions of high activation temperature of widespread practical interest small departures of the Lewis number from unity are often found to have appreciable effects on flamelet behavior (Sivashinsky, 1976; and Buckmaster and Mikolaitis, 1982). Therefore, it is of interest to extend previous results to Lewis numbers different from unity. Such an extension is reported here.

The present study differs, from earlier work concerned with the influence of nonunity Lewis numbers in that the full range of rates of strain a flamelet can experience from low to moderate to high is considered along with the effects of variable density associated with heat release. The analysis of Sivashinsky (1976) is restricted to weak rates of strain and to constant density flows. That of Buckmaster and Mikolaitis (1982), also restricted to constant density flows, considers conditions herein termed 
low-to-moderate rates of strain but sometimes termed strong strain by others; under these strain conditions a thin reactive-diffusive zone exists on the product side of a convective-diffusive zone with the separation between the stagnation point and the former less than or of the same order as the thickness of the latter. In addition to these regimes we also consider conditions of high rates of strain under which the reaction zone no longer is thin; these conditions are identified by Liñán and Crespo (1976) but appear not to have been discussed for flows with Lewis numbers different from unity. Because of different boundary conditions the present two-stream problem differs from the problem of one stream of reactants incident on an adiabatic wall, which has been considered by a number of investigators.

All of the previous studies cited thus far involve the constant density approximation. Treatments of the full fluid mechanics of flamelets are only beginning to appear. In addition to LWI and LWII the work of Clavin and Williams (1982) falls in this category. Although restricted to low rates of strain, its predictions of flame temperature and flame speed can be compared with those of a limiting case of the present analysis.

All of these studies indicate that the effects of variable density on flamelet behavior are substantial. For example, Buckmaster and Mikolaitis (1982) find that adiabatic flamelets with constant density and Lewis numbers exceeding unity readily experience abrupt transitions associated with extinction. We show here the same flamelets with density variations of practical interest require unrealistically large deviations of the Lewis number from unity for such transitions to occur.

As discussed in LWI and LWII motivations for the present work include interest in ascertaining the structures and dynamics of flamelets in turbulent flames under conditions of both weak and intense turbulence and in utilizing observations on the extinction characteristics of laminar flames in the laboratory to determine effective chemical kinetic behavior. The former consideration motivates the work of Clavin and Williams (1982) and the latter the work of Buckmaster and Mikolaitis (1982). These last authors conjecture on the basis of their earlier work that unless the reactivediffusive zone lies on the reactant side of the stagnation point the flame will be extinguished. This conjecture, of course, is not precisely consistent with solutions having that zone located somewhat on the product side of the stagnation point and requires either thorough stability analyses that are difficult to perform or careful experimental investigations for its assessment.

\section{FORMULATION}

The counterflowing streams of reactants and products are shown schematically in Figure 1 along with the coordinates and key variables used in their characterization. When the equations of LWII are extended to include general values of the Lewis number $L e$, they become

$$
\begin{gathered}
f^{\prime \prime}+f f^{\prime \prime}+\frac{1}{2}\left[1+\tau(H+c)-f^{\prime 2}\right]=0 \\
c^{\prime \prime}+\sigma L e f c^{\prime}=D[1+\tau(H+c)](1-c) \exp \left\{-T_{a} /[1+\tau(H+c)]\right\} \\
H^{\prime \prime}+\sigma f H^{\prime}-\left(\frac{1}{L e}-1\right) c^{\prime \prime}=0
\end{gathered}
$$

subject to the following boundary conditions: 


$$
\begin{aligned}
f(0) & =0 \\
f^{\prime}(\infty) & =1, \quad c(\infty)=H(\infty)=0 \\
f^{\prime}(-\infty) & =\left[1+\tau\left(H_{\infty}+1\right)\right]^{1 / 2}, \quad c(-\infty)=1, \quad H(-\infty)=H_{\infty}
\end{aligned}
$$

In these equations the independent variable is the usual similarity variable $\eta$ involving the rate of strain $a$ and the density ratio $\rho=[1+\tau(H+c)]^{-1}$ with the value unity in the reactant stream. The dependent variables $f, c$ and $H$ are respectively the

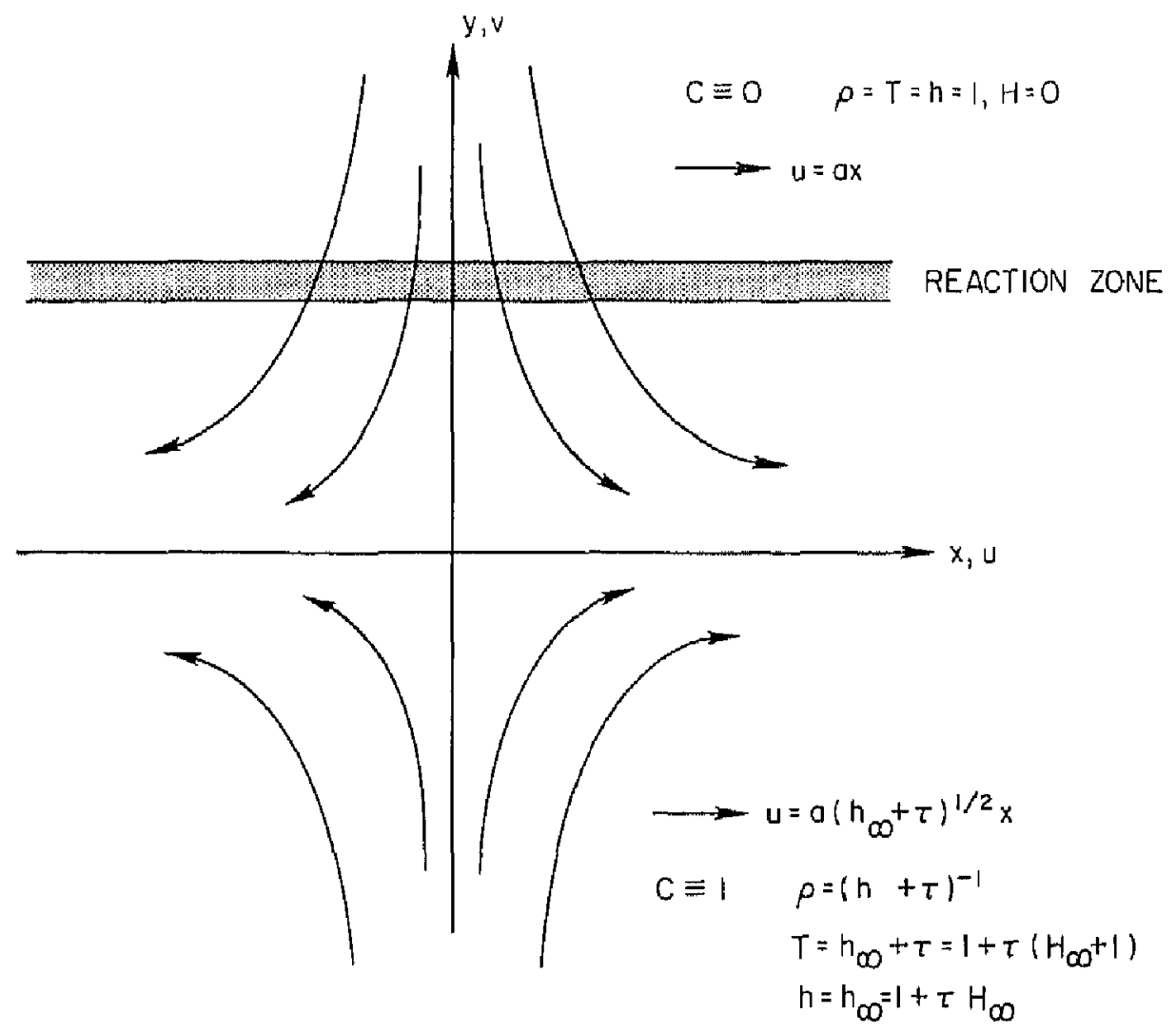

FIGURE 1 Schematic representation of the flow (from $\mathbf{L W I I ) .}$

modified stream function yielding the velocity components, e.g., $u=a x f^{\prime}(\eta)$; the progress variable indicating the extent of the chemical reaction; and a modified enthalpy function such that $h=1+\tau H$ where $h$ is the usual static enthalpy normalized to have the value unity in the reactant stream. The parameter $\tau$ indicates the degree of heat release and is related to the physical observable temperature ratio in the product stream by the equation $T_{\infty}=1+i\left(H_{\infty}+1\right)$ where $T$ is unity in the reactant stream. In general $T=1-\tau(H+c)$, a physically significant relation since it is the influence of nonunity Lewis numbers on the temperature which combines with large activation temperatures to yield the appreciable effects on flamelet behavior cited earlier. The parameters $H_{\infty}$ and $h_{\infty}$ each determine the enthalpy in the product stream and both are used in the presentation of numerical results. Finally, the Damköhler number $D=B \sigma L e / 2 p_{0} a$ where $B$ is a constant preexponential factor. We maintain our previous point of view and consider flamelets subject to various rates of strain $a$ and 
thus involving various Damköhler numbers as a consequence of variations in those rates. The term proportional to $c^{\prime \prime}$ in Eq. (3) shows that both nonadiabaticity and nonunity Lewis numbers alter the enthalpy and thus the temperature.

In applying AEA to these equations the small expansion parameter always involves the reciprocal of the activation temperature $T_{a}$ but takes on various forms depending on the case being considered.

We follow the sequence of studies in LWII and deal first with flamelets subject to low-to-moderate rates of strain. In this case chemical reaction is confined to a thin reaction zone involving the usual diffusive-reactive balance. A double limiting case of nearly adiabatic flows with Lewis numbers nearly equal to unity is considered first and is found to expose in a relatively simple fashion the principal features of this study. Next we consider the more general case of nonadiabatic flows involving gases with Lewis numbers near unity and demonstrate the influence of arbitrarily large deviations from adiabaticity and of small deviations of the Lewis number from unity. Finally, we take up the case of flamelets subject to such high rates of strain that reactions occurs far into the product stream in extended reaction zones involving a diffusive-convective-reactive balance.

\section{NEARLY ADIABATIC FLOWS WITH LEWIS NUMBERS NEAR UNITY}

It is convenient to eliminate the Lewis number in favor of an alternative parameter $\psi=L e^{-1}-1$ which takes on small values when the Lewis number is near unity; note that $\psi>0$ if $L e<1$ and $\psi<0$ if $L e>1$. We now consider a double expansion for nearly adiabatic flows involving gases with Lewis numbers nearly unity, i.e., for $H_{\infty}, \psi \ll 1$. When these expansions are combined with the expansion associated with AEA, i.e., with an appropriate $\varepsilon \rightarrow 0$, both $H_{\infty}$ and $\psi$ will in due course be required to approach zero in appropriate fashions.

The definitions of $\varepsilon$ and of the Damköhler parameter $\Delta$ used in LWII for the nearly adiabatic case are applicable here and we therefore take:

$$
\begin{aligned}
D \exp \left[-\frac{T_{a}}{1+\tau(H+c)}\right] & =\frac{\Delta}{\varepsilon^{2}} \exp \left\{\frac{1}{\varepsilon \tau}\left[1-\frac{1+\tau}{1+\tau(H+c)}\right]\right\} \\
\frac{1}{\varepsilon \tau}-2 \ln \varepsilon & =\ln D-\ln \Delta \\
\varepsilon \tau T_{a} & =1+\tau
\end{aligned}
$$

As noted in LWII the parameter $\varepsilon$ defined by the last of these equations is fundamental since it is known provided only that the physically relevant parameters $T_{a}$ and $\tau$ are known. Alternative expansion parameters will arise later in other cases and will not possess this feature. Also note that the Damköhler parameter $\Delta$ is an $O(1)$ quantity; thus the validity of numerical results leading to values of $\Delta$ large or small compared to unity must be questioned. $\dagger$

$\dagger$ A distinction must be made between the limit analysis and numerical results based on that analysis. The Damköhler parameter is independent of the limit process but when small, nonzero values of $\varepsilon$ are selected in order to develop numerical results, then questions of validity arise if in the course of the calculations, large or small values of $\Delta$ are encountered. 
With $H \ll 1$ and $\varepsilon \rightarrow 0$ the first of these equations indicates that chemical reaction is confined to a zone where $c \rightarrow 1$. We locate this zone at $\eta=\eta_{r}$ and as in LWI and LWII we find first the outer solutions with $\eta_{r}$ specified. Subsequently, the matching of the inner and outer solutions determines a relation between $\Delta$ and $7_{r}$ and thus the rate of strain resulting in the specified reaction zone location.

\subsection{The Outer Solutions}

Only the outer solutions to lowest order in $\varepsilon$ are needed for this case and these are obtained in terms of two regular expansions with $H_{\infty}$ and $\psi$ as parameters. Thus we assume

$$
\begin{aligned}
& f=f_{0}(\eta)+H_{\infty} f_{H 1}(\eta)+\psi f_{\psi 1}(\eta)+\ldots \\
& c=c_{0}(\eta)+H_{\infty} c_{H 1}(\eta)+\psi c_{\psi / 1}(\eta)+\ldots \\
& H=H_{\infty} H_{H 1}(\eta)+\psi H_{\psi / 1}+\ldots
\end{aligned}
$$

Substitution of these equations into Eqs. (1)-(2) leads to lowest order in the expansion parameters to

$$
\begin{aligned}
f_{0}{ }^{\prime \prime}+f_{0} f_{0}{ }^{\prime \prime}+\frac{1}{2}\left(1+\tau c_{0}-f_{0}{ }^{\prime 2}\right) & =0 \\
c_{0}{ }^{\prime \prime}+\sigma f_{0} c_{0}{ }^{\prime} & =0, \quad \eta>\eta_{r} \\
c_{0} & =1, \quad \eta<\eta_{r}
\end{aligned}
$$

The solutions of these equations yield the distributions of the velocity components and of the progress variable in adiabatic flamelets with Lewis number equal to unity and are given in LWI and LWII.

Substitution of Eqs. (6) into Eq. (3) results in two equations, one for $H_{H 1}(\eta)$ and the other for $H_{\psi 1}(\eta)$; we obtain

$$
H_{H 1}^{\prime \prime}+\sigma f_{0} H_{H 1}^{\prime}=0
$$

with boundary conditions

$$
H_{H 1}(\infty)=0, \quad H_{H 1}(-\infty)=1
$$

and

$$
\begin{aligned}
H^{\prime \prime}{ }^{\prime}+\sigma f_{0} H^{\prime}{ }_{\psi 1} & =c^{\prime \prime}{ }_{0}, \quad \eta>\eta_{r} \\
& =0, \quad \eta<\eta_{r}
\end{aligned}
$$

with boundary conditions

$$
\begin{aligned}
& H_{\psi 1,1}(\infty)=H_{\psi 1}(-\infty)=0 \\
& H_{\psi 1}\left(\eta_{r}-\right)=H_{\psi 1}\left(\eta_{r}+\right)
\end{aligned}
$$

Equations (8) and (9) determine the perturbed enthalpies. The solutions for $H_{H I 1}$ are 
continuous in all derivatives but those for $H_{\psi 1}$ are discontinuous in the first derivative at $\eta=\eta_{r}$; from $\mathrm{Eq}$. (9) it is easily determined that the final boundary condition is

$$
H_{\psi^{\prime} 1}^{\prime}\left(\eta_{r^{+}}\right)=H_{\psi 1}^{\prime}\left(\eta_{r}^{-}\right)+c^{\prime}{ }_{0 r}
$$

where $c^{\prime} 0 r \equiv c^{\prime} 0\left(\eta_{r}{ }^{+}\right)$and where in general the subscript $r$ denotes a value at the reaction zone location $\eta=\eta_{r}$.

The solutions to Eqs. (7)-(9) can be obtained numerically and provide values needed for subsequent developments, namely $f_{0 r}, H_{H 1 r}, H_{H 1 r}^{\prime}, H_{\psi 1}, H_{\psi r 1}^{\prime}\left(\eta_{r^{-}}\right)$, and $H_{\psi 1}^{\prime}\left(\eta_{r}{ }^{+}\right)$.

It will be useful later to consider the behavior of $H_{\psi 1} 1 r(\eta)$ as $\eta_{r \rightarrow \infty}$. In this case the solution of Eq. (9) for $\eta<\eta_{r}$ must satisfy approximately the alternative boundary condition $H_{\psi 1}(\eta \rightarrow \infty) \rightarrow H_{y / 1}$ so that $H_{\psi 1}^{\prime}\left(\eta_{r^{-}}\right) \approx 0$. Thus Eq. (10) yields $H_{\psi \psi^{\prime} 1}^{\prime}\left(\eta_{r}{ }^{+}\right) \approx c^{\prime}{ }^{\prime}$. The solution for $\eta>\eta_{r}$ can be found analytically; we are justified to let $f_{0} \approx \eta+\kappa$ where $\kappa$ is a constant given by the outer solutions for adiabatic flows. Furthermore, from LWI and LWII we know that under these circumstances

$$
c_{0} \approx \frac{\eta_{r}+\kappa}{\eta+\kappa} \frac{\exp \left(-\frac{\sigma}{2}(\eta+\kappa)^{2}\right)}{\exp \left(-\frac{\sigma}{2}\left(\eta_{r}+\kappa\right)^{2}\right)}
$$

so that

$$
-c^{\prime}{ }_{0 r} \approx\left(\eta_{r}+\kappa\right)\left[\sigma+\left(\eta_{r}+\kappa\right)^{-2}+\ldots\right]
$$

With these approximations for $f_{0}$ and $c_{0} \dagger$ it can be shown by the use of Eq. (9) that

$$
H_{\psi 12}=\frac{1}{\sigma\left(\eta_{r}+\kappa\right)^{2}}+\ldots
$$

We thus see that as $\eta_{r} \rightarrow \infty H_{\psi 1 r} \rightarrow 0$ but at an algebraic rate in contrast with $H_{H 1 r}$ which decays to zero exponentially fast. The implication of these different asymptotic behaviors is that the effect of nonunity Lewis numbers is more significant than nonadiabaticity as the rate of strain decreases.

At the other limit as $\eta_{r} \rightarrow-\infty\left(-c^{\prime}{ }_{0 r}\right) \rightarrow 0$ so it is clear that then $H_{\psi 11} \rightarrow 0$. It simplifies our exposition if we anticipate our numerical results and note that $H_{\psi 11 r}>0$ over the entire range of $\eta_{r}$.

\subsection{Inner Solutions}

The coordinate stretch necessary to resolve the structure of the reaction zone is given in LWII, namely

$$
\xi=\frac{\eta-\eta_{r}}{\varepsilon}\left[\frac{-c^{\prime}{ }_{0 r}}{1+\tau}\right]
$$

The requirement suggested by Eq. (13), namely that $\left(-c^{\prime}{ }^{\prime}\right.$ ) $)$ not be too small, restricts

tNote that in Eq. (11) $k=k\left(\eta_{t}\right)$. 
our considerations to rates of strain termed low-to-moderate. As indicated earlier a different analysis is called for when the rates of strain are so high as to make $\left(-c^{\prime}{ }_{0 r}\right)$ unsuitably small for Eq. (13) to apply.

For the resolution of the structure of the reaction zone the progress variable is expressed as

$$
c=1-\varepsilon(1+r) y_{1}(\xi)+\ldots
$$

Use of these equations in Eq. (2) and retention of only the lowest order terms in $\varepsilon$ leads to

$$
y_{1}^{\prime \prime}=\left\{\frac{\Delta(1+\tau)^{3} \exp \left[\frac{1}{(1+\tau)}\left(x H_{H 1 r}+\chi_{L} H_{\psi 1 r}\right)\right]}{\left(-c_{0 r}{ }^{\prime}\right)^{2}}\right\} \times y_{1} \exp \left(-y_{1}\right)
$$

where $\chi=H_{\infty} / \varepsilon$ is the 0 (1) parameter introduced in LWII arising from the double limit $H_{\infty}, \varepsilon \rightarrow 0$ and where $X_{L}=\psi / \varepsilon$ is the corresponding parameter arising from the double limit $\psi, \varepsilon \rightarrow 0 . \dagger$ The left side of Eq. (14) comes from the diffusion term of Eq. (2), the right side from the chemical term so that we have the usual diffusivereactive balance within the reaction zone.

Equation (14) is frequently encountered in AEA. Standard procedures involving matching of the inner and outer solutions establish that the factor within the brackets on the right side equals $1 / 2$. Thus we have the following equation for the determination of $\Delta$ :

$$
\Delta=\frac{\left(-c^{\prime}{ }^{\prime}\right)^{2}}{2(1+\tau)^{3}} \exp \left[-\frac{1}{(1+\tau)}\left(\chi H_{H 1 r}+\chi_{L} H_{\psi 1 r}\right)\right]
$$

It is worth noting two variants of Eq. (15); if $H_{\infty}=0$, i.e., if the flamelet is adiabatic, we have

$$
\Delta=\Delta_{a} \exp \left(-\chi_{L} \frac{H_{\psi 1} 1 r}{1+\tau}\right)
$$

Since $H_{\psi 1 r}>0$, we see from this equation that $\Delta>\Delta_{a}$, i.e., the rate of strain for a given reaction zone location is reduced, if $X_{L}<0$, i.e., e.g., if $\psi<0, L e>1$. The opposite change occurs if $X_{L}>0$.

For $\eta_{r} \gg 1$ from Eq. (12) we find that the temperature at the reaction zone, i.e. , the flame temperature, is

$$
T_{r}=1+\tau+\frac{\tau(1-L e)}{L e \sigma\left(\eta_{r}+\kappa\right)^{2}}
$$

The last term in this expression describes the departure from the adiabatic flame temperature and by use of Eqs. (11), (15) and (16) may be shown to be $[\sigma \tau(1-L e)] /$

$\dagger$ Note that $\chi$ and $\chi_{L}$ are independent of the limit process $\varepsilon \rightarrow 0$. However, in numerical results involving specific, small but nonzero values of $\varepsilon$ the absolute values of these parameters are limited by the linearization associated with the expansions in $H_{i s}$ and $\psi$. 
$\left[2 L e(1+\tau)^{3} \Delta_{u}\right]$ for $H_{x}=0$. Here the factor Le in the denominator is unity to the order of accuracy of the calculation. When the results of Clavin and Williams (1982) for the flame temperature are expressed in the present notation the comparable expression is

$$
\left[\sigma(1-L e) / 2(1+\tau)^{3} \Delta_{a}\right] \int_{0}^{\tau}(1 / x) \ln (1+x) d x
$$

The difference in the two expressions only involves the $\tau$-dependence and is traceable to their assumption of $\mu$ constant while we take $\rho \mu$ constant. The present results may be shown to agree with those of the more recent work of Clavin and Garcia (1983) when their general results are specialized to the case of $\rho \mu$ constant.

Equation (15) can also be rewritten in the form of a generalization of Eq. (16), namely as

$$
\Delta=\Delta_{L e=1} \exp \left(-\chi_{L} \frac{H_{\psi 1 r}}{1+\tau}\right)
$$

In this form our earlier discussion of Eq. (16) is seen to apply more generally to nearly adaibatic flamelets. Equation (17) will be of interest for purposes of comparison with the results of the next section wherein general nonadiabatic flamelets are treated.

\subsection{Extrema in the Damköhler Parameter}

From LWII and similar applications of AEA we know that extrema in $\Delta$ can be related to the extinction and ignition behavior of flamelets. In fact in choosing values of the relatively large number of parameters which arise in this study we emphasize those especially relevant to such behavior. Here we examine the possibility of extrema determined by Eq. (15). In doing so we hold $\chi_{\text {and }} \chi_{L}$ fixed and set to zero the partial derivative $\partial \Delta / \partial \eta_{r}$. We find

$$
\begin{aligned}
\left(\chi+\chi_{L} \frac{\partial H_{\psi 1} / \partial \eta_{r}}{\partial H_{H 1 r} / \partial \eta_{r}}\right)_{e} & =\frac{2(1+r)}{c^{\prime}{ }_{0 r}} \frac{\partial c^{\prime}{ }_{0 r} / \partial \eta_{r}}{\partial H_{H 1 r} / \partial \eta_{r}} \\
& =\chi_{e, L e=1}
\end{aligned}
$$

where $\chi_{e, L e=1}<0$ depend on $\eta_{r}$ and $\tau$ and are the values of $\chi$ leading to extrema in $\Delta$ for nearly adiabatic flamelets with unity Lewis number, values given in LWII. The negative values of $\chi_{e, L e=1}$ imply that cooling of the product stream is called for if such extrema are to arise under these circumstances. For given values of $\eta_{r}$ and $\tau$ both the quotient of the partial derivatives on the left side and $\chi_{e, L e=1}$ are known so that Eq. (18) defines a linear relation between $\chi_{e}$ and $\chi_{L e}$, the values of these parameters resulting in extrema in $\Delta$ for the specified $\eta_{r}$ and $\tau$. For a given value of the latter the various lines for the complete range of $\eta_{r}$ form an envelop which divides the $\chi_{e}-\chi_{L e^{-}}$ plane into two regions: One for pairs of values of $\chi_{e}$ and $\chi_{L e}$ resulting in monotonic variations of $\Delta$ with $\eta_{r}$ and a second resulting in multiple values of $\eta_{r}$ for a range of values of $\Delta$.

Consider the derivatives appearing in Eq. (18). In LWII analytic approximations for two of these derivatives are presented, approximations which have the advantage of being given by quantities determined by the outer solutions and which are based on the 
neglect of the variation with $\eta_{r}$ of the function $f\left(\eta ; \eta_{r}\right)$ for the purposes of calculating the desired derivatives. We have

$$
\begin{aligned}
& \frac{\partial}{\partial \eta_{r}}\left(c_{0 r}^{\prime}\right)=-\sigma f_{0 r} c^{\prime}-\left(c^{\prime} 0 r\right)^{2} \\
& \frac{\partial}{\partial \eta_{r}} H_{H 1 r}=H_{H 1 r}^{\prime}
\end{aligned}
$$

The calculation of the derivative $\partial H_{\psi 1 \gamma} / \partial \eta_{r}$ can be carried out in a similar fashion so that the following approximation is obtained:

$$
\frac{\partial}{\partial \eta_{r}} H_{\psi 1 r}=H_{\psi 1}^{\prime}\left(\eta_{r}^{-}\right)+\frac{\left(-c^{\prime}{ }_{0 r}\right)\left[H_{\psi 1}^{\prime}\left(\eta_{r}+\right)+\left(-c_{0 r}^{\prime}\right)\left(1+H_{\psi 1 r}\right)\right]+\partial c^{\prime}{ }_{0 r} / \partial \eta_{r}}{\left(-c^{\prime}{ }_{0 r}\right)+\left(H_{\psi^{\prime} 1}^{\prime}\left(\eta_{r}-\right) / H_{\psi r 1}\right)}
$$

Note that these equations require little calculation once the outer solutions are available.

In the present study we have carried out the exact determination of the derivatives appearing in Eq. (18). In the Appendix we develop expressions depending on perturbations relative to $\eta_{r}$; of particular interest are the functions $\hat{c}\left(\eta ; \eta_{r}\right), \hat{H}_{1}\left(\eta ; \eta_{r}\right)$ and $\hat{H}_{2}\left(\eta ; \eta_{r}\right)$. When these are calculated numerically, the derivatives in question are given by the following equations:

$$
\begin{aligned}
& \frac{\partial c^{\prime}{ }_{0 r}}{\partial \eta_{r}}=-\sigma f_{0 r} c_{0 r}^{\prime}+c^{\prime}{ }_{0 r} \hat{c}^{\prime}\left(\eta_{r} ; \eta_{r}\right) \\
& \frac{\partial H_{H 1 r}}{\partial \eta_{r}}=H^{\prime}{ }_{H 1 r}+c^{\prime}{ }_{0 r} \hat{H}_{1}\left(\eta_{r} ; \eta_{r}\right) \\
& \frac{\partial H_{\psi 1 r}}{\partial \eta_{r}}=H_{\psi 1}^{\prime}{ }_{\psi 1}\left(\eta_{r}{ }^{+} ; \eta_{r}\right)+c^{\prime}{ }_{0 r} \hat{H}_{2}\left(\eta_{r}+; \eta_{r}\right)
\end{aligned}
$$

It is not possible to make even qualitative assessments of the accuracy of the approximate expressions for these derivatives but the numerical results establish that the latter two derivatives are sufficiently accurate for most purposes. The least accurate is the derivative $\partial c^{\prime}{ }^{\prime} / \partial \eta_{r}$; for $\eta_{r}<0$ and for positive values in the neighborhood of the stagnation point the approximate expressions are accurate within 10 percent but as $\eta_{r}$ increases the approximation degrades until for $\tau=3$ it is in error by 40 percent as $\eta_{r} \rightarrow \infty$. This is precisely the same error given by Eq. (11) if the variation of $\kappa$ with $\eta_{r}$ is not taken into account. We thus conclude that for many purposes the analytic approximations given by Eqs. (19) and (20) are adequate.

\subsection{Rate of Creation of Product}

In LWI and LWII we define and use the parameter $m$, the rate of creation of product for arbitrary rates of strain normalized to that for the corresponding unstrained flame, as a measure of the rate at which fresh product is created. For the conditions in this 
section we can immediately extend the development in LWII (cf. Eq. (19) in LWII) and obtain

$$
m=\left[\frac{\Delta_{a}}{\Delta}\right]^{1 / 2}=\exp \left[\frac{1}{2(1+\tau)}\left(\chi_{H 1 r}+\chi_{L} H_{\psi / 1 r}\right)\right]
$$

From Eq. (21) we can infer the limiting behavior of the rate of creation of product in the limits $\eta_{r} \rightarrow \pm \infty$; as $\eta_{r} \rightarrow \infty m \rightarrow 1$ but with $H_{\psi r 1 r}$ dominating the asymptotic behavior. With $H_{\psi 11}>0$ the approach of $m \rightarrow 1$ depends in this limit on the sign of $\chi_{L}$. For $\eta_{r} \rightarrow-\infty m \rightarrow \exp [x / 2(1+\tau)]$ and $H_{H 1 r}$ dominates.

\subsection{Results for Nearly Adiabatic Flamelets with Lewis Numbers Near Unity}

The distributions of outer solutions related to the case considered in this section except those pertaining to $L e \neq 1$ are given in LWI and LWII. Those for $H_{\psi 1}(\eta)$ and $\hat{H}_{2}(\eta)$ are interesting and are therefore given in Figures 2 and 3 for unit Prandtl number, for $\tau=3$ and for various reaction zone locations. Before discussing these results it is useful to note that two effects contribute to $H_{\psi 1}(\eta)$; first, the effect of diffusion, differences in the rates of diffusion of heat and reactant, as manifest in the $c_{0}{ }^{\prime \prime}$-term is operative for all reaction zone locations including those corresponding to $\eta_{r} \gg 1$. The discontinuity in $H^{\prime}{ }_{\psi 1}^{1}$ is a manifestation of chemical reaction but its effect on $H_{\psi t}(\eta)$ depends on the reaction zone location, becoming increasingly large as $\eta_{r} \rightarrow \infty$ and becoming vanishingly small as $\eta_{r} \rightarrow-\infty$.

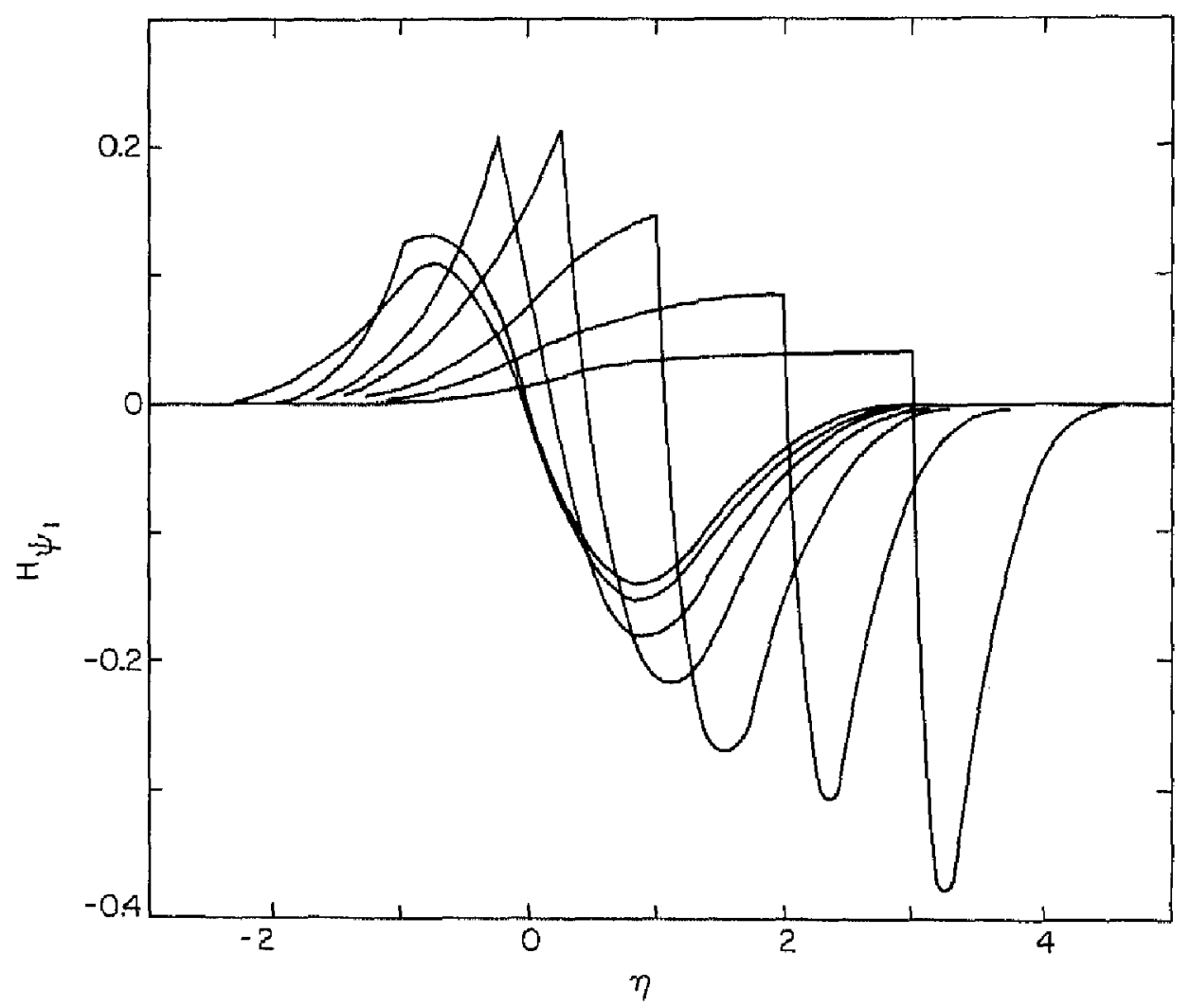

FIGURE 2 The perturbed enthalpy distributions: $\tau=3$. 


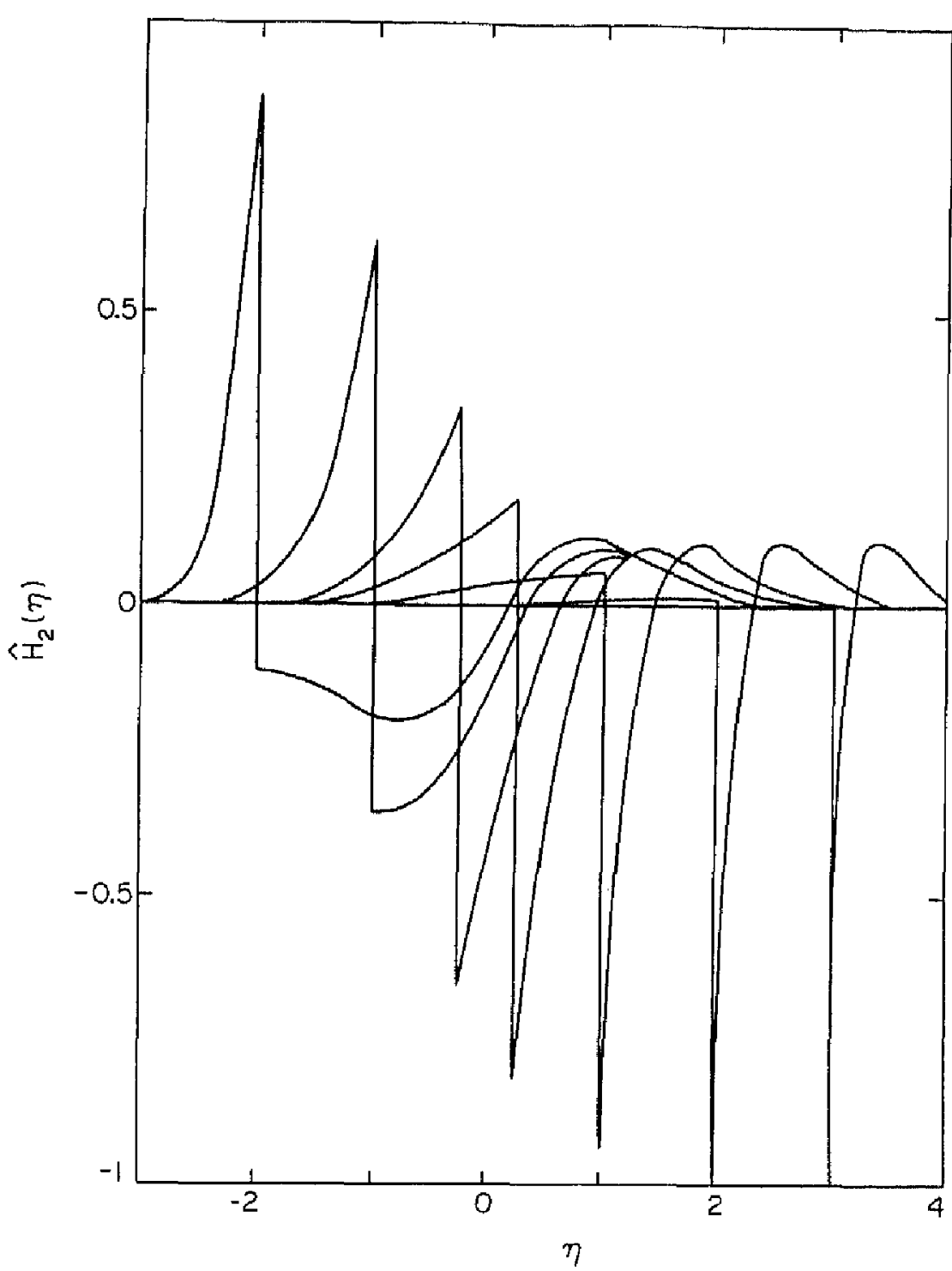

FIGURE 3 Contribution to the derivative of the perturbed enthalpy with respect to the reaction zone location: $\tau=3$.

These effects lead to the interesting distributions shown in Figure 2. For $\eta_{r} \gg 1 H_{\gamma_{r} 1}\left(\eta<\eta_{r}\right)$ displays the behavior leading to Eq. (12) but $H_{\psi r}\left(\eta>\eta_{r}\right)$ exhibits a complex variation due to the large value of $H^{\prime}{ }_{\psi 1}\left(\eta_{r}{ }^{+}\right)$and to the large values of $c^{\prime \prime}{ }_{0}$. It is the influence of these two effects which results in the algebraic decay of $H_{\psi 1 \mathrm{t}}$ as $\eta_{r} \rightarrow \infty$. For $-\eta_{r} \gg 1$ the effect of the discontinuity in $H^{\prime}{ }_{\psi 1}$ at the reaction zone is so small that $H_{\psi t 1}(\eta)$ is nearly continuous. The curves in Figure 3 are influenced by the same phenomena that influence $H_{\psi 1}$ but are more complex because of the discontinuities expressed by Eqs. (A-7) and (A-8).

Because the significantly different behavior of $H_{F I 1 r}$ and $H_{\psi 11 r}$ plays an important role in determining flamelet characteristics, we show in Figures $4 \mathrm{a}$ and $4 \mathrm{~b}$ their distributions with reaction zone location for $\tau=0,3$ and 6 . The perturbation associated with near adiabaticity decreases monotonically from unity to zero as $\eta_{r}$ increases from minus to plus infinity. In contrast the complex behavior of $H_{\psi 1}(\eta)$ shown for $\tau=3$ in Figure 2 leads to the distributions of $H_{\psi / r}$ shown in Figure $4 \mathrm{~b}$. We see that $H_{\gamma^{\prime} 1 r}>0$ as $\eta_{r} \rightarrow \pm \infty$ has significant implications for the effect indicated earlier and that a maximum 


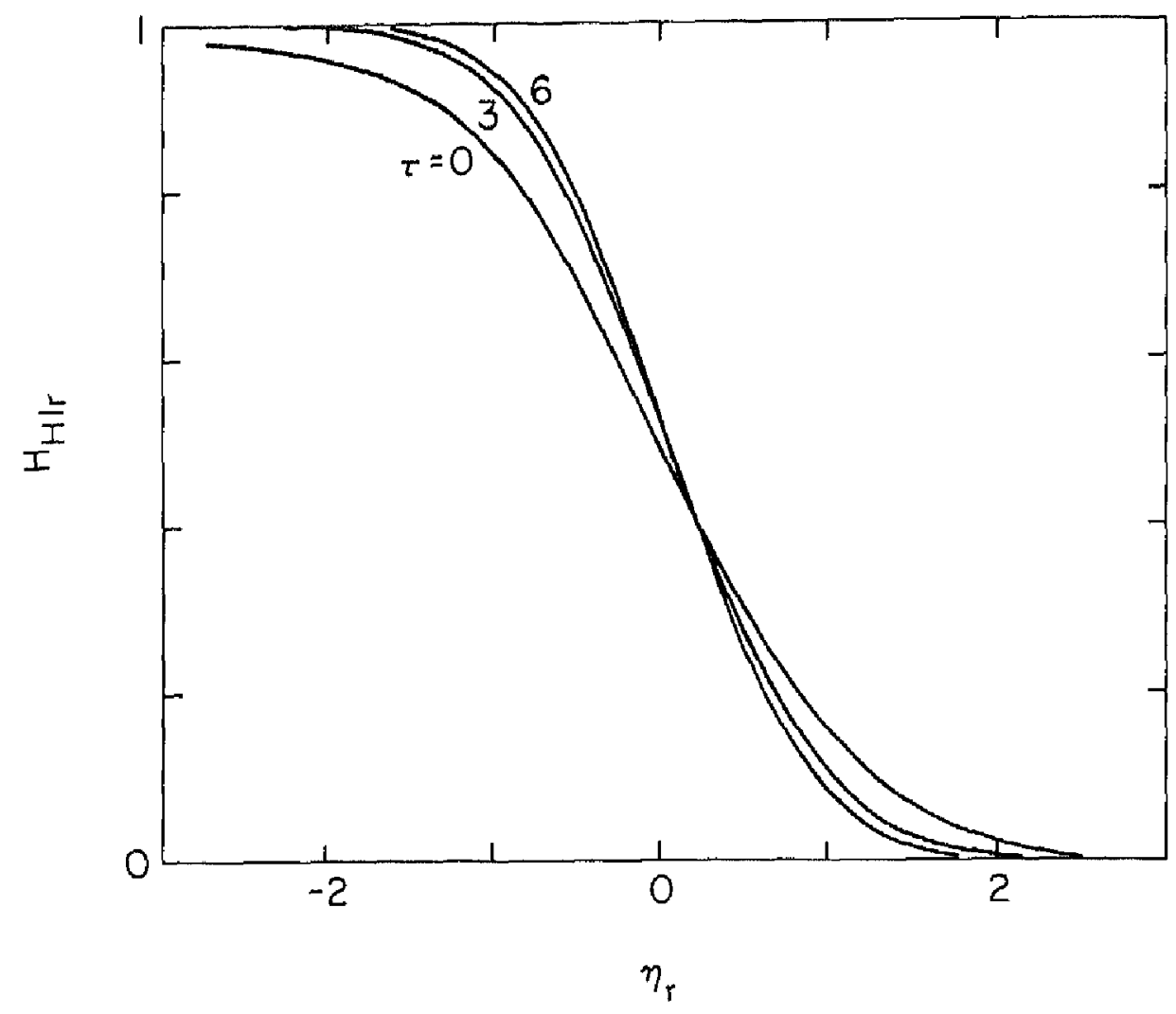

FIGURE 4 The perturbed enthalpies at the reaction zone. (a) The nearly adiabatic perturbation $H_{H 1 r}$ (b) The near unity Lewis number perturbation $H_{p 1 r}$.

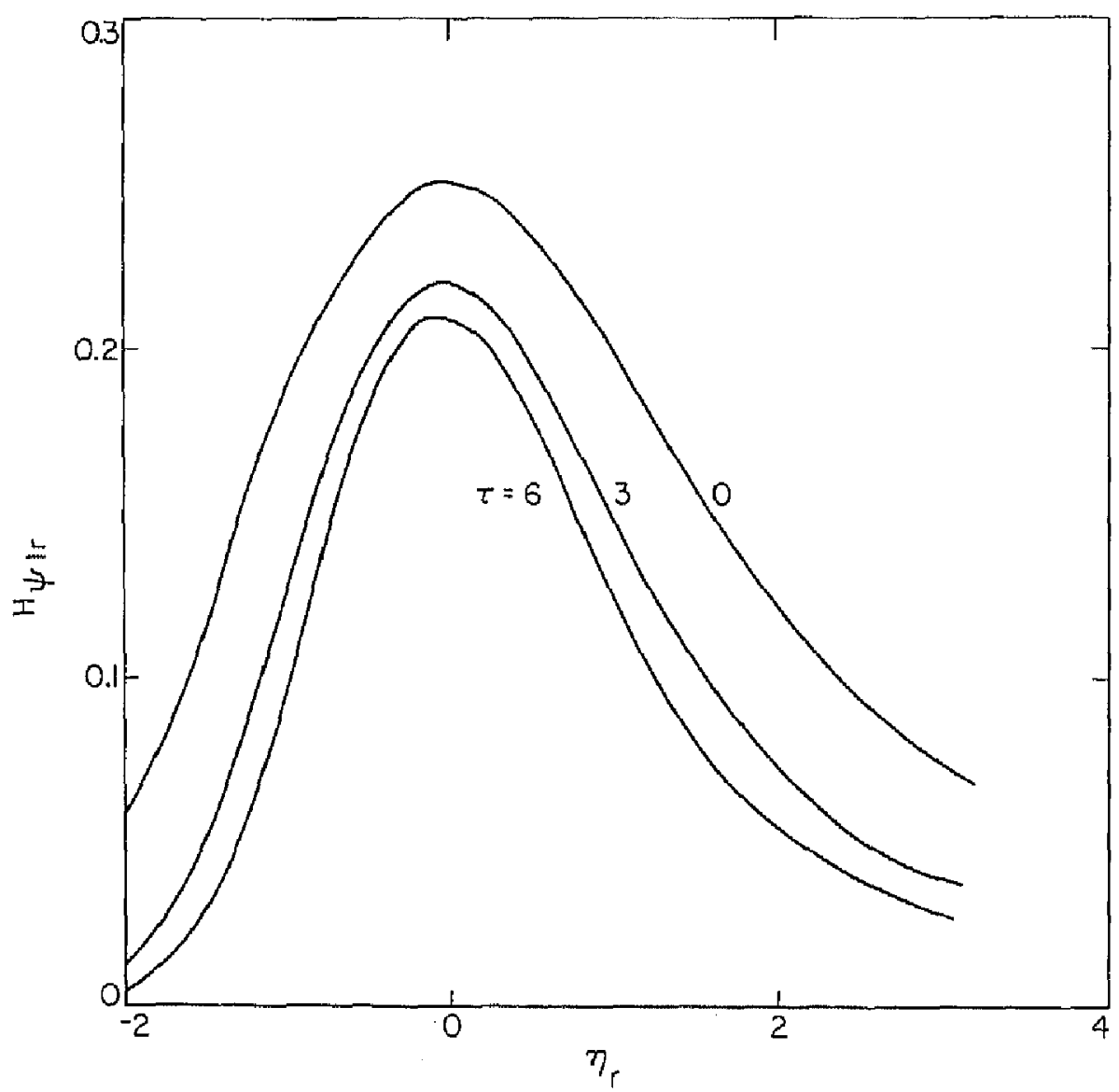


occurs when the reaction zone is in the neighborhood of the stagnation point. We can anticipate from Eq. (18) that the vanishing of $\partial H_{\gamma / r} / \partial \eta_{r}$ has significant implications with respect to the dependence on $\eta_{r}$ of the effect of nonunity Lewis numbers on flamelet behavior.

We develop this implication further and show in Figure 5 the variation of $\chi_{L e}$ with $\eta_{r}$ for $\chi=0$, i.e., the variation of the values of $\chi_{L}$ resulting in extrema of $\Delta$ under adiabatic conditions. Note that we display $\chi_{L e}$ for $\eta_{r}<0$ and $-\chi_{L e}$ for $\eta_{r}>0$. To

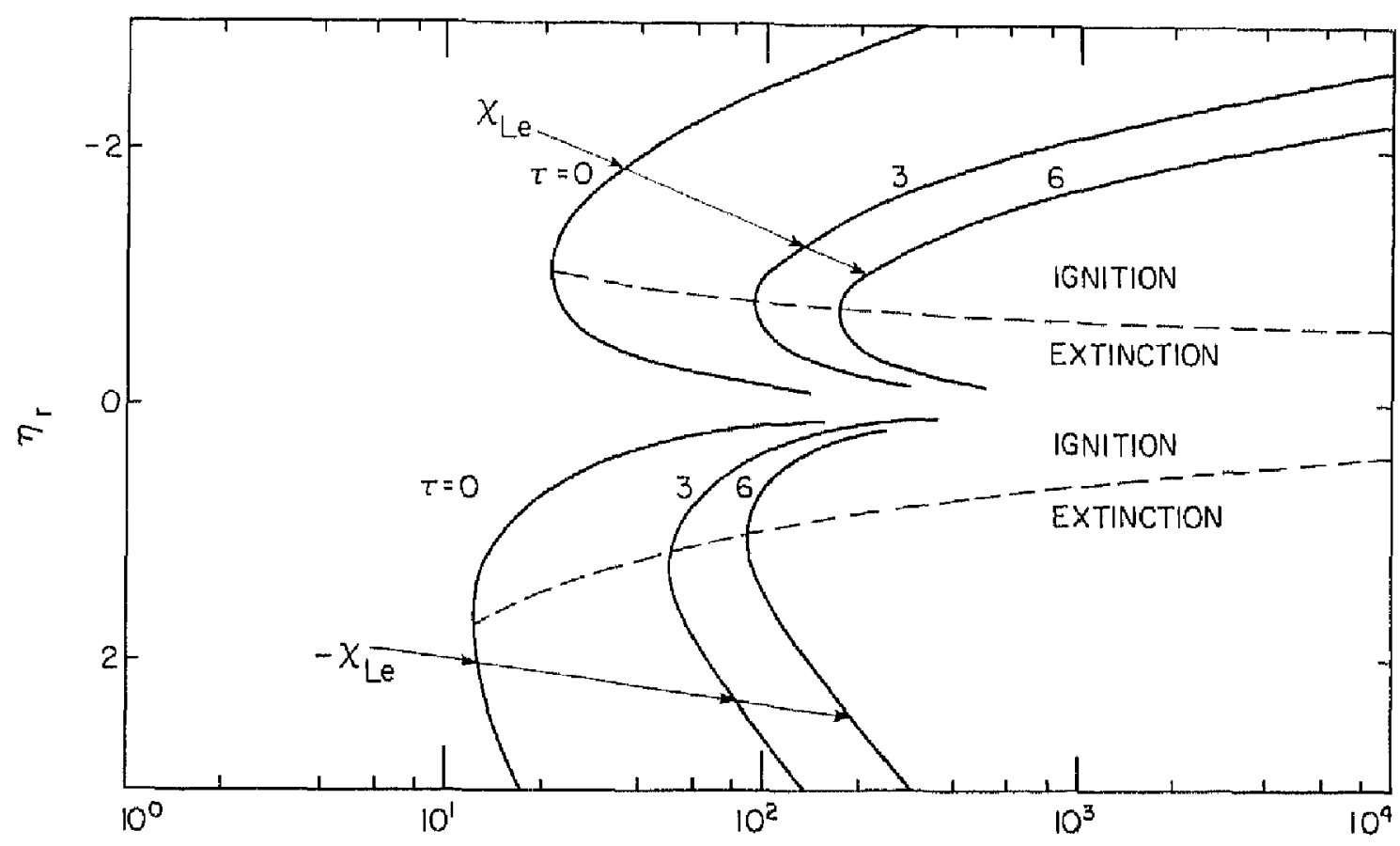

FIGURE 5 The variation of $\chi_{L e}$ with reaction zone location.

focus the discussion consider the results for $\tau=3$; we see that for $-50<\chi_{L e}<90$ there are no extrema in $\Delta$ implying that relatively large absolute values of $\chi_{L} \equiv \psi / \varepsilon$ are required if adiabatic flamelets are to encounter the abrupt transitions in reaction zone location and in the rate of creation of product shown in LWII to arise in nearly adiabatic and nonadiabatic flamelets with unity Lewis number. As we shall see the distributions for $\eta_{r}>0$ correspond to those usually associated with extinction and ignition. However, those for $\eta_{r}<0$ may not be of practical significance since the values of $\chi_{L e}$ are impractically large for values of $\varepsilon$ of practical in interest. $\dagger$ Nevertheless, we show in Figure 6 the distributions of $\Delta$ with $\eta_{r}$ for $r=3$ and for representative values of $\chi_{L}$. The resulting distributions are reminiscent of the S-curves found in LWII for nearly adiabatic and nonadiabatic flamelets with unity Lewis number and in other applications of AEA (cf. Williams 1971). For $-\chi_{L}<50 \Delta$ increases monotonically with increases in $\eta_{r}$ implying that the reaction zone assumes positions toward the reactant stream as the rate of strain takes on smaller values. For $-\chi_{L}>50$, however, extrema in $\Delta$ arise and abrupt transitions in reaction zone location can occur. Note from Figure 6 that as $\eta_{r} \rightarrow \infty$ the approach of $\Delta$ toward $\Delta_{a}$ is relatively slow, a consequence of the algebraic decay of $H_{\psi 1}$ discussed earlier.

TNote that if $\chi_{L}=O\left(10^{2}\right)$ and if the linearization associated with the expansion in powers of $\psi$ is to be respected, then $\varepsilon=O\left(10^{-3}\right)$ which is too small to be of practical significance. 


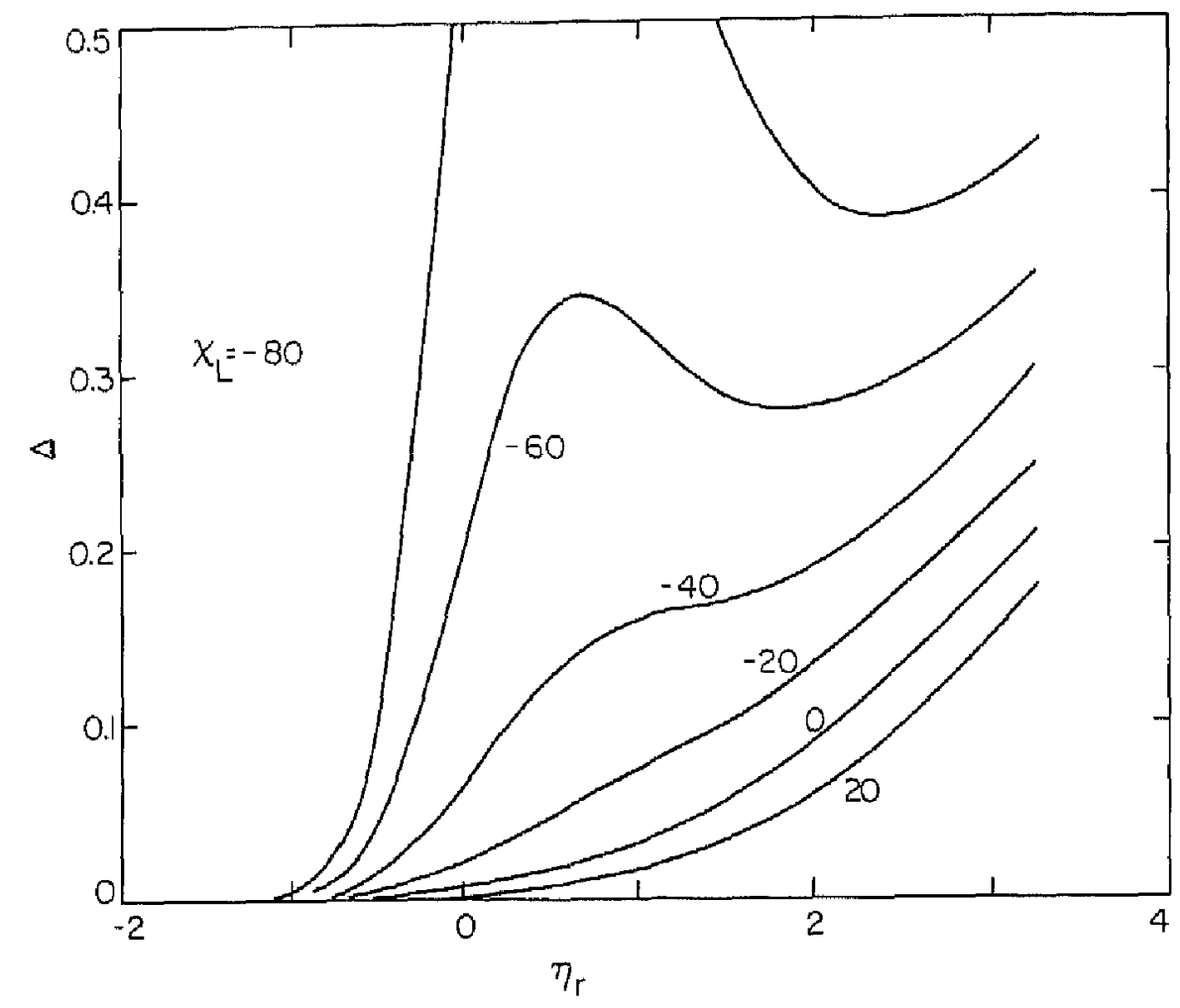

FIGURE 6 Variation of the Damköhler parameter with reaction zone location for adiabatic flamelets: $\tau=3$.

Figure 7 gives the variation with $\Delta$ of the rate of creation of product as reflected in the parameter $m$ for the values of $\chi_{L_{4}}$ used in Figure 6. These distributions resemble those for nearly adiabatic flamelets with unity Lewis number given in LWII. The monotonic variations on Figure 6 correspond to similar variations of $m$ in Figure 7, whereas the variations with multiple reaction zone locations for a given $\Delta$ lead to

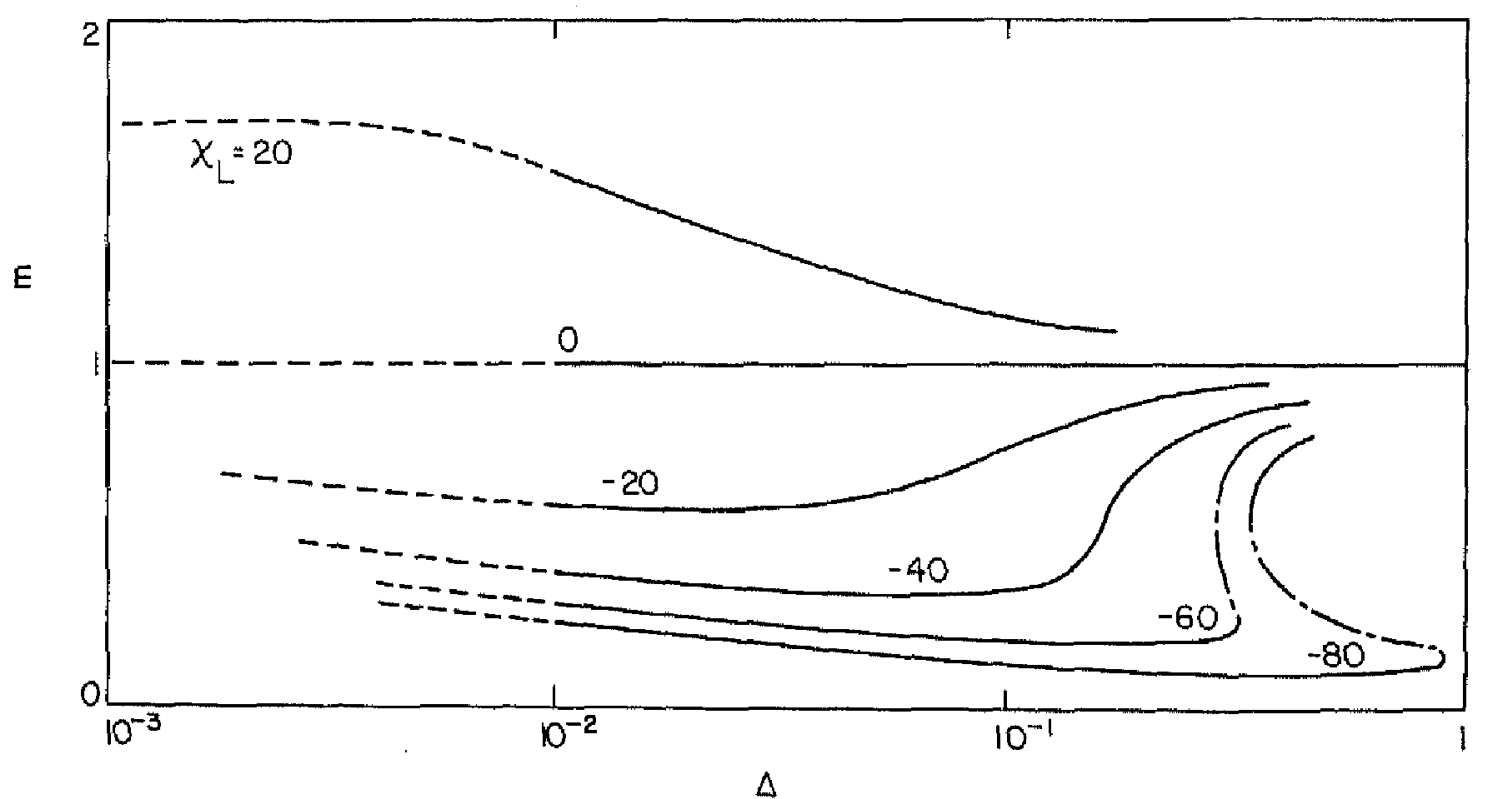

FIGURE 7 Variation of the parameter related to the mass rate of creation of product with the Damköhler parameter for adiabatic flamelets: $\tau=3$. 
multiple values of $m$. Although we know from our earlier discussion [cf. Eqs. (12) and (21)] that $m \rightarrow 1$ as $\Delta$ increases without limit, we see from Figure 7 that the algebraic decay of $H_{\psi 1 r}$ leads to a slow approach of the rate of creation of product to that of an unstrained flamelet. Note that for $\Delta<10^{-2}$ we continue the curves as dashed lines to suggest an uncertainty as to their validity when interpreted in terms of small, nonzero values of $\varepsilon$.

The implications of Figures 6 and 7 can be understood most readily if a famelet involving specified thermochemical parameters is imagined to be subject to a sequence of rates of strain. Consider, for example, a low rate of strain sequentially incremented to larger values. If $-X_{L}<50$, the reaction zone assumes a corresponding continuous sequence of locations closer to and then farther from the stagnation point on the side of the product stream. We shall find later that a decrease in the rate of creation of product accompanies this sequence. If $-\chi_{L}>50$, the continuous sequence of reaction zone locations is interrupted when the minimum in $\Delta$ is reached. The interruption involves an abrupt transition of reaction zone location to one usually in the product stream. Again we shall see later that this transition involves a significant reduction in the rate of product creation and thus a partial extinction.

Similar considerations apply when flamelets are subject to high but decreasing rates of strain. In this case when $-\chi_{L}>50$, the abrupt transition occurs at the maximum in $\Delta$ and involves a significant increase in the rate of creation of product and thus an ignition.

An interesting variant can be obtained from Figure 5 by use of results such as those in Figure 6. We show in Figure 8 a replot of the results such as those given in Figures 5 and 6, namely a graph of $\Delta$ versus $\chi_{L e}$. In contrast with Figure 5 we see that Figure 8 displays a cusp behavior. The point of the cusp corresponds to the minimum of the continuous curve of Figure 5 while the two sides of the cusp correspond to conditions for extinction and ignition. The cusp shape is a consequence of the nature of the dependence of $\Delta$ on $\eta r$; it is interesting that such cusps represent one of the known singularities of catastrophe theory. There is a similar cusp in the dependence of $\Delta$ on $\chi_{e}$ in the results of LWII although a curve corresponding to Figure 8 is not given there.

Two cusps are shown in Figure 8, one for the portion of Figure 5 with $\chi_{L e}<0$ and the other for that with $\chi_{L e}>0$. The former is realistic with values of $\Delta$ of order unity while the latter has $\Delta$ of order $10^{-5}$ or less, values which cast doubt on its physical significance.

The signs of $\chi_{L e}$ in Figures 5 and 8 are explicable in terms of the influence of strain on flame temperatures with $L e \neq 1$, i.e., the temperature at the reaction zone. Consider negative values of $\chi_{L}, i, e ., L e>1$; a positive strain rate is known to tend to reduce the flame temperature by enhancing the diffusion of heat relative to that of reactant. Such reduced temperatures are responsible for the extinction transition at sufficiently large rates of strain seen for $x_{L}<-50$.

The situation for rates of strain resulting in $\eta_{r}<0$ is somewhat more complicated. First, it should be noted that for $\chi_{L}>0$, i.e., for $L e<1$, increases in the rate of strain tend to increase the flame temperature, again through the competition between diffusion of heat and reactant. This results in an increase in the rate of consumption of reactant with increasing $\Delta$ as seen, for example, in Figure 7 for $\chi_{L}=20$. However, when $\Delta$ becomes very small, i.e, when the rates of strain are very large (and the values of $\eta_{r}$ are negative) the flame temperature which under these circumstances is greater than the adiabatic value begins to decrease toward the temperature of the product stream and $m$ begins to decrease toward unity as seen in Figure 7. The decrease in flame temperature with increasing strain rate at high values thereof can lead to abrupt 


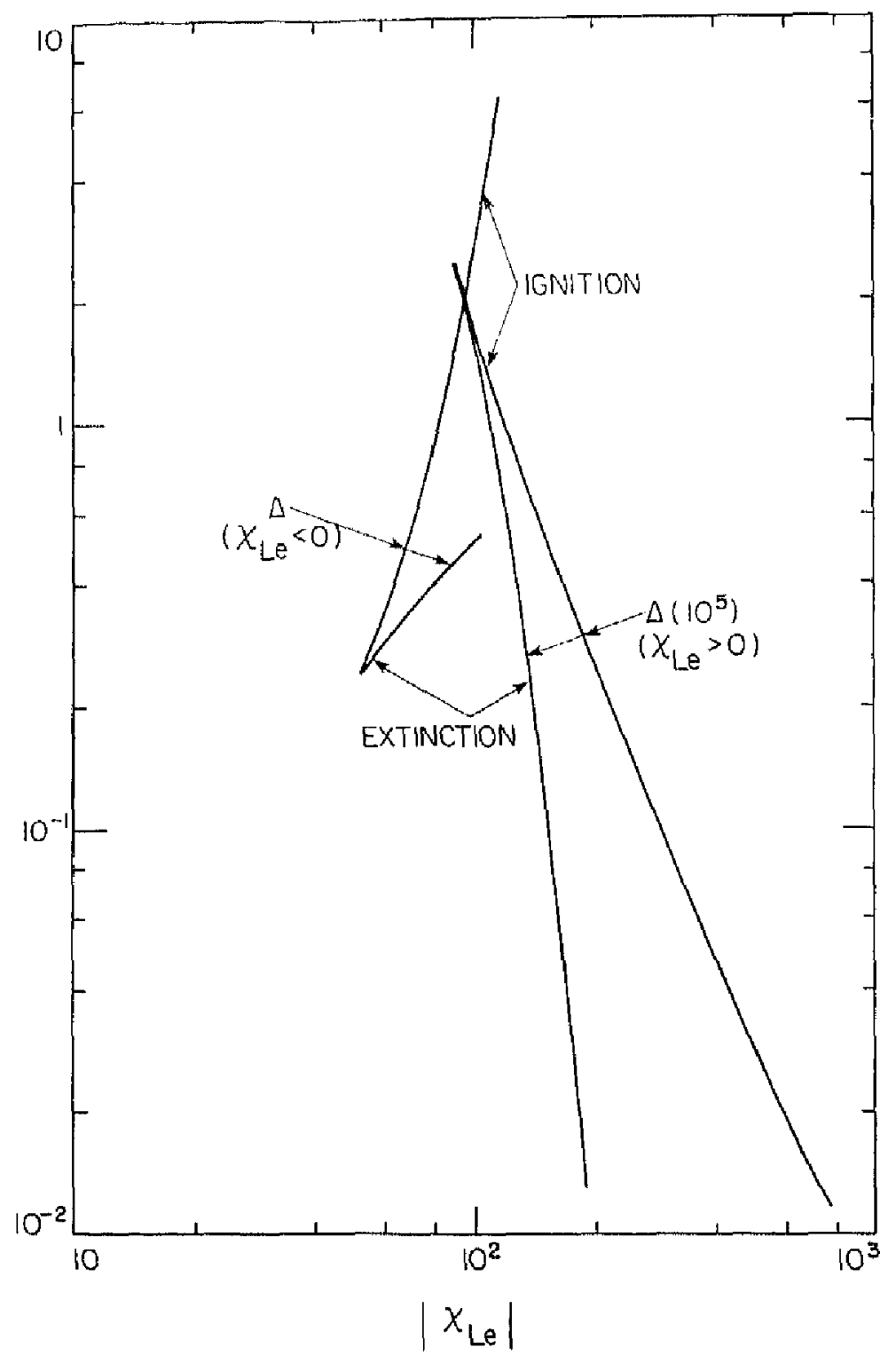

FIGURE 8 Variation of the Damköhler parameter $\triangle$ at the extrema versus $\chi_{L e}: \tau=3$.

extinction when $\Delta$ becomes sufficiently small and when $\chi_{L}$ is sufficiently large $\left(\chi_{L}>90\right.$ for $\tau=3$ as indicated in Figure 5 for $\eta_{r}<0$ and in Figure 8 for $\chi_{L e}>0$ ). Although the aerothermochemistry of this transition is clear, its practical significance is in question because it is predicted to occur only at unusually large values of $\chi_{L}$ and small values of $\Delta$.

We now discuss the characteristics of nearly adiabatic flamelets with near unity Lewis numbers. The values of $\chi$ we adopt for the numerical results are suggested by values of $H_{\infty}$ and $\varepsilon$ used in the next section while for $x_{L}$ we assume values of 20 and -40 to represent the influence of Lewis numbers less than and greater than unity respectively although these values are relatively extreme from a practical point of view. As an introductory remark it is worth recalling our earlier observation that the variations of $H_{H 1 r}$ and $H_{\psi 1 r}$ in the limits $\eta_{r} \rightarrow \pm \infty$ are significantly different and thus that we should expect nonunity Lewis number effects to be dominant over nonadiabatic effects only for $\eta_{r} \gg 1$, i.e., only for low rates of strain.

We first show in Figure 9 the combinations of $\chi_{e}$ and $\chi_{L e}$ resulting in single- and 
multiple-values of $\eta_{r}$ for a range of $\Delta$. In this figure the intercepts on the $\chi_{c^{\text {-axis }}}$ correspond to $X_{e, L e=1}$ and are given in LWII while those on the $X_{L e}$-axis are shown in another form in Figure 5. Note the compressed scale of the $X_{I, c}$-axis implying that (in comparison with nonadiabaticity) relatively large deviations of the Lewis number from unity are required to achieve multiple-valuedness.

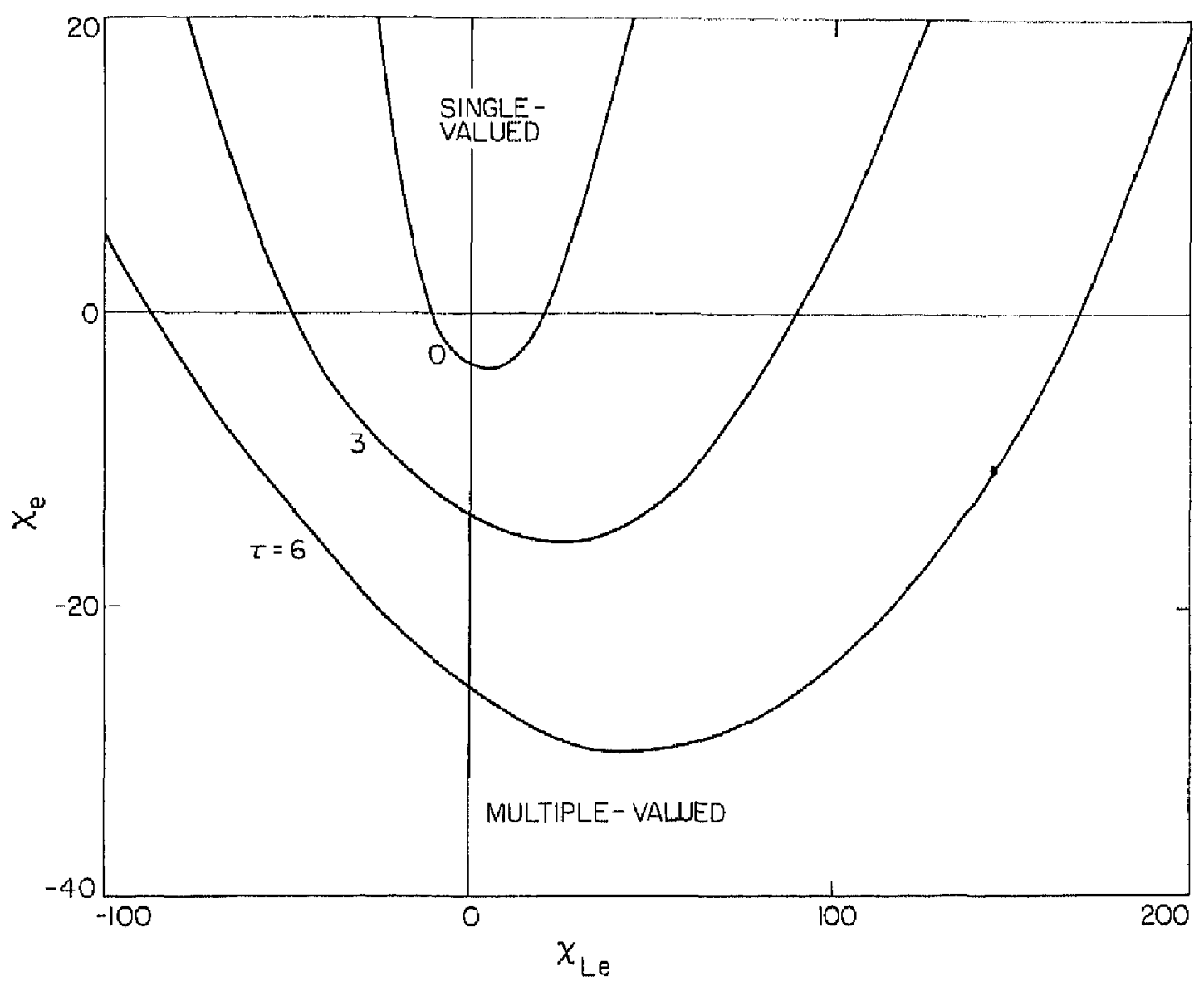

FIGURE 9 The boundaries of the single- and multiple-valued distributions of the Damköhler parameter $\Delta$.

Figures $10 \mathrm{a}$ and 1 la relate to nearly adiabatic flamelets with Lewis numbers slightly less than unity while Figures $10 \mathrm{~b}$ and $11 \mathrm{~b}$ do likewise for Lewis numbers slightly greater than unity. Consider first Figures 10a and 10b; if these are compared with the corresponding figure in LWII for $\chi_{L}=0$ or with one another, it is clear that greater degrees of product cooling $(-\chi$ larger) are required for ffa melets with Lewis numbers slightly less than unity to encounter multiple reaction zone locations for a given rate of strain. Alternatively, we can interpret these figures as indicating that flamelets with Lewis numbers slightly greater than unity are more likely to involve abrupt transitions in reaction zone location and the consequent abrupt changes in the rate of creation of product. Also to be noted from Figures $10 \mathrm{a}$ and $10 \mathrm{~b}$ is the persistence of the influence of nonunity Lewis numbers for low rates of strain.

The corresponding variations of the rate of creation of product as reflected in the parameter $m$ are given in Figures 11a and 11b. It is useful to discuss these results in terms of flamelets subjected to a sequence of decreasing rates of strain with an initial large value. From Figure 11a which relates to Lewis numbers slightly less than unity we see that for slightly superadiabatic flamelets $(x>0)$ the values of $m$ decrease 


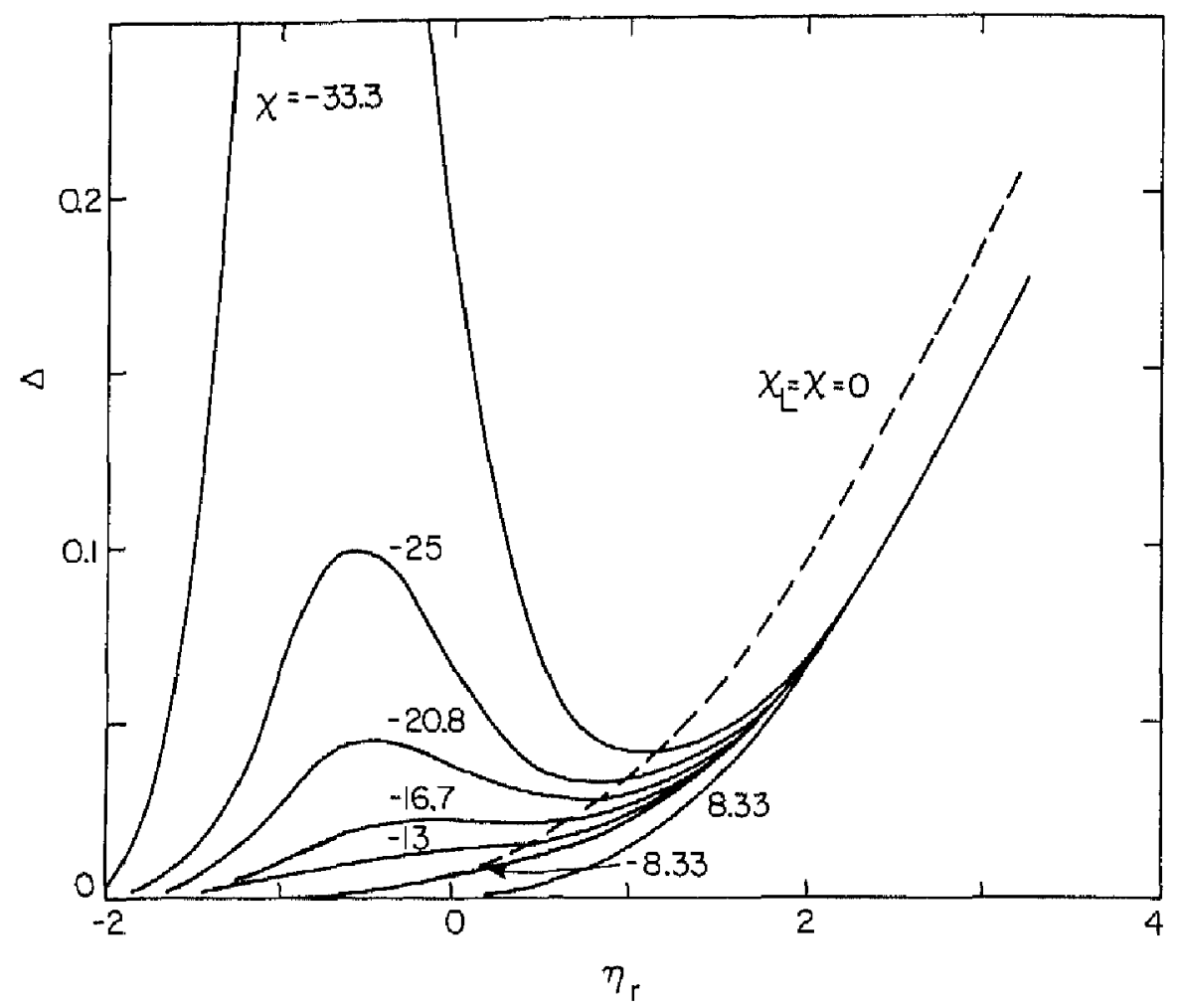

FIGURE 10 Variation of the Damköhler parameter with reaction zone location for nearly adiabatic flamelets with near unity Lewis numbers: $\tau=3$. (a) $\chi_{L}=20$, (b) $\chi_{L}=-40$.

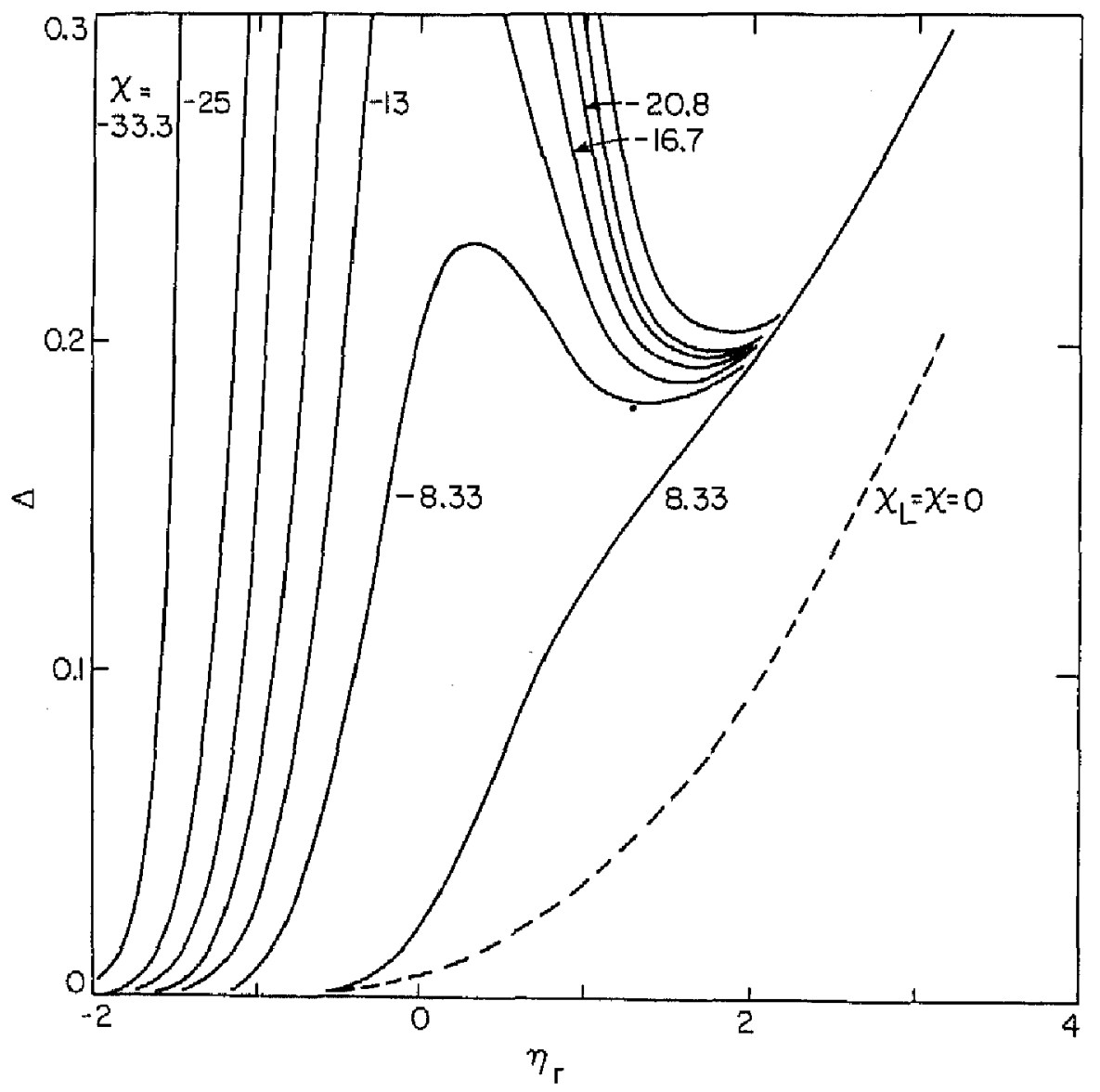




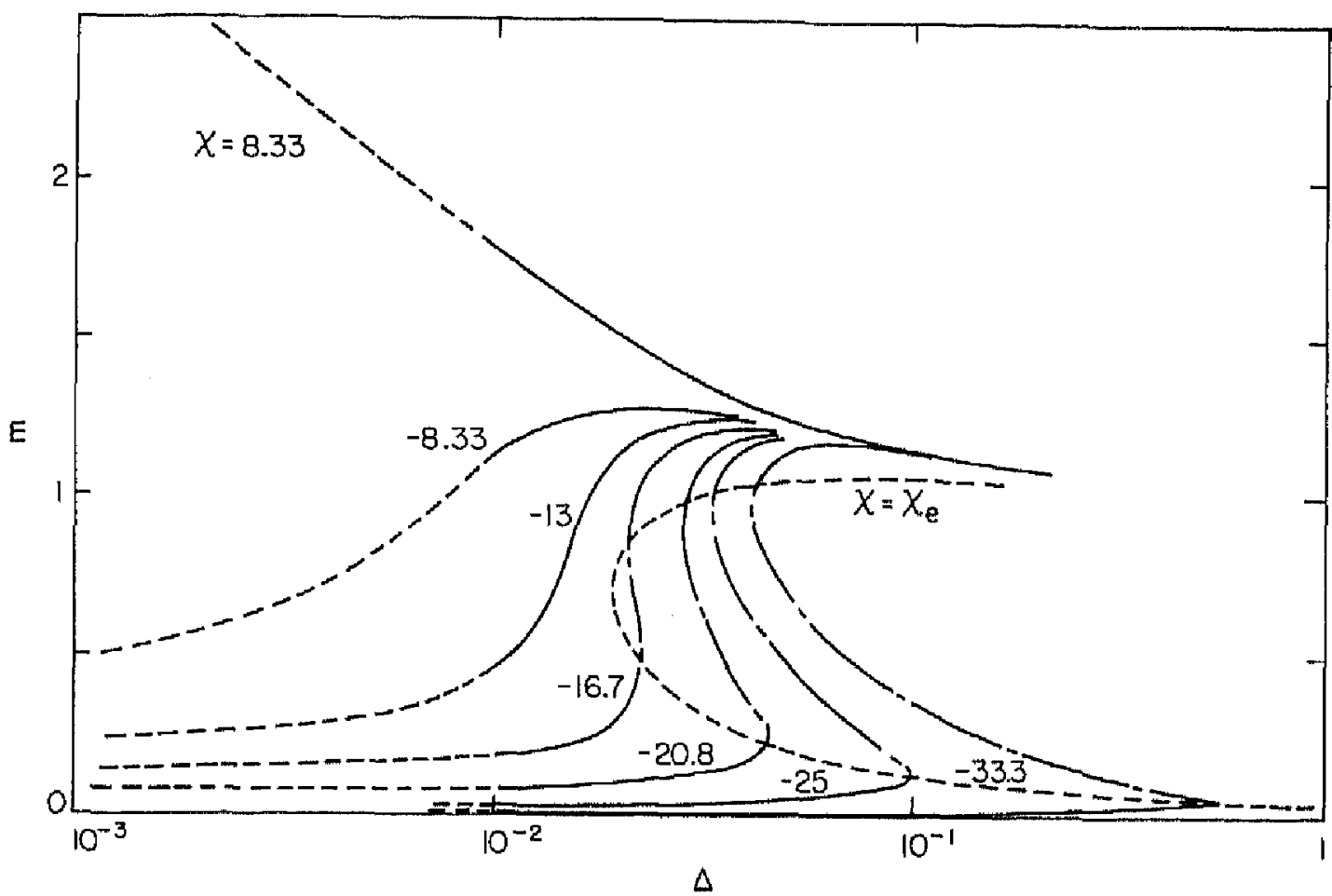

FIGURE 11 Variation of the parameter for the mass rate of creation of product with the Damköhler parameter for nearly adiabatic flamelets with near unity Lewis numbers: $\tau=3$ (a) $\chi_{L}$ $=20$, (b) $x_{L}=-40$.

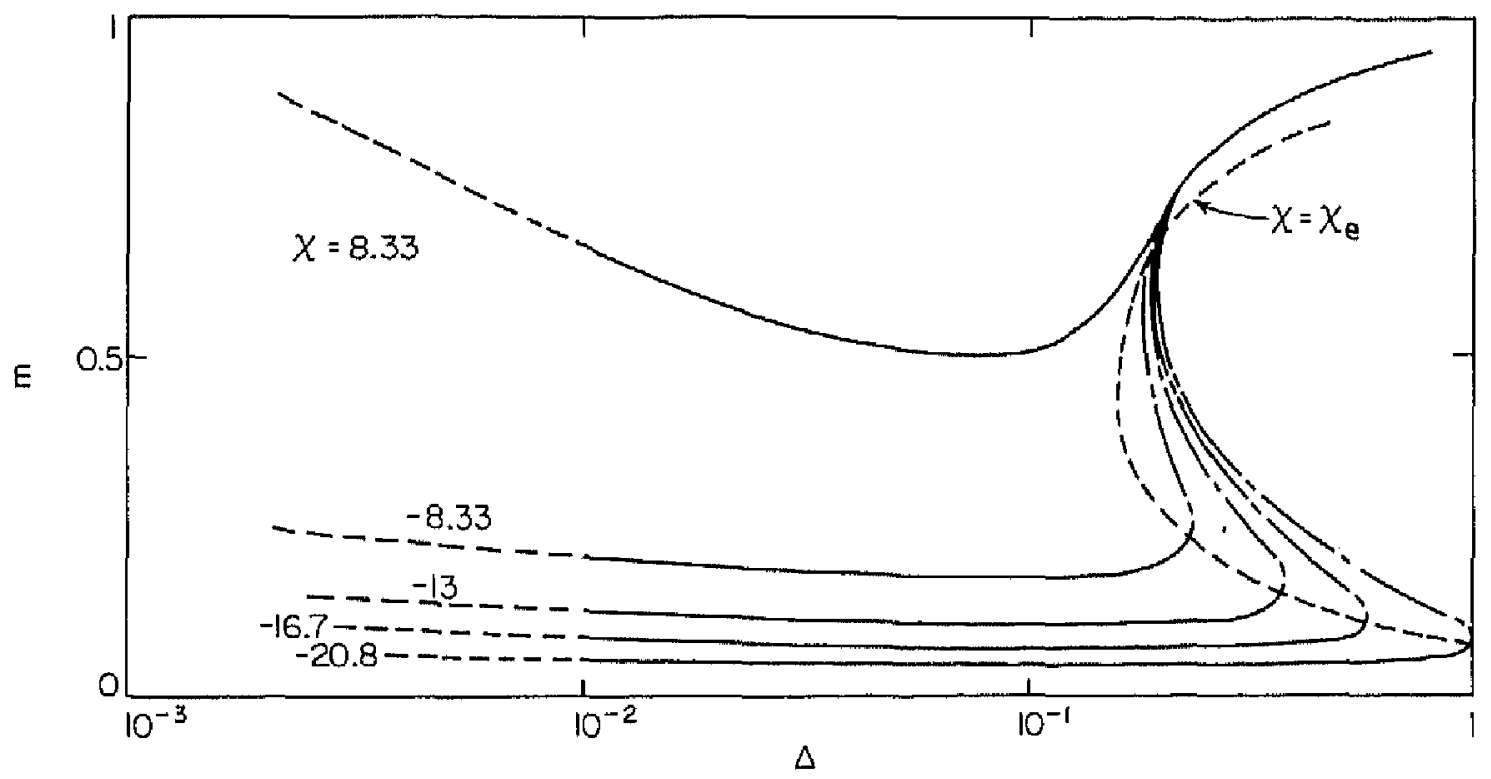

monotonically toward unity in the range shown. In contrast subadiabatic flamelets with degrees of cooling sufficiently small so that $-\chi>15$ involve continuous increases in $m$ followed by a maximum as the rate of strain decreases. However, for greater degrees of product cooling the abrupt transitions in reaction zone location suggested by Figure 10a yield abrupt increases in the rate of creation of product to values of $m$ that usually exceed unity. Further decreases in the rate of strain cause $m$ to approach unity from above. 
Qualitatively the same behavior is shown in Figure $1 \mathrm{lb}$ for flamelets with Lewis numbers slightly greater than unity. In this case the approach of $m$ toward unity as the rate of strain decreases is from values less than unity so that even for slight superadiabaticity and large rates of strain $m<1$.

\section{NONADIABATIC FLAMELETS WITH LEWIS NUMBERS NEAR UNITY}

We now turn our attention to flamelets with arbitrarily heated or cooled product streams but with Lewis numbers near unity. The strategy of solution in this case is the same as in the previous section; we obtain outer solutions with $\eta_{r}$ as a parameter subsequently related to the Damköhler number and thus to the related rate of strain via the inner solutions. However, here we need both the first and second order outer solutions in an expansion parameter representing an appropriate generalization of $\varepsilon$. The second order solutions in $\psi$ introduce another parameter which is subsequently determined by matching the inner and outer solutions.

The extension of Eqs. (5) to account for general nonadiabaticity is given in LWIl, namely by

$$
\begin{aligned}
D \exp \left[-\frac{T_{a}}{1+\tau(H+c)}\right] & =\frac{\Delta_{n}}{\varepsilon_{n}{ }^{2}} \exp \left[\frac{1}{\varepsilon_{n} \tau}\left\{1-\frac{h_{0 r}+\tau}{1+\tau(H+c)}\right\}\right] \\
\frac{1}{\varepsilon_{n} \tau}-2 \ln \varepsilon_{n} & =\ln D-\ln \Delta_{n} \\
\varepsilon_{n} \tau T_{a} & =h_{0 r}+\tau
\end{aligned}
$$

where $h_{0 r}$ is an enthalpy parameter defined later. In Eqs. (22) we add the subscript $n$ to $\varepsilon$ and $\Delta$ to distinguish them from their counterparts in the previous section.

The comments in LWII regarding interpretation of results in terms of $\varepsilon_{n}$ and $\Delta_{n}$ and the importance of extrema in $\Delta$ rather than in $\Delta_{n}$ for determining extinction and ignition characteristics apply here. Thus we repeatedly use the following equations to relate $\varepsilon_{n}$ and $\Delta_{n}$ of this section to the more fundamental parameters $\varepsilon$ and $\Delta$ :

$$
\begin{aligned}
\varepsilon_{n} & =\varepsilon\left[\frac{h_{0 r}+\tau}{1+\tau}\right] \\
\Delta_{n} & =\Delta\left[\frac{h_{0 r}+\tau}{1+\tau}\right]^{2} \exp \left[\frac{1}{\varepsilon \tau}\left\{\frac{h_{0 r}-1}{h_{0 r}+\tau}\right\}\right]
\end{aligned}
$$

We see that the Damköhler parameters are related only if either $\varepsilon_{n}$ or $\varepsilon$ is specified, an unfortunate necessity.

\subsection{The Outer Solutions}

The outer solutions are obtained from Eqs. (1)-(3) by means of a double expansion in $\varepsilon_{n}$ and $\psi$. For simplicity we consider only the requisite functions, minimize the subscripts without loss of clarity and thus let 


$$
\begin{aligned}
f\left(\eta ; \varepsilon_{n}, \psi\right)= & {\left[f_{0}(\eta)+\psi f_{01}(\eta)+\ldots\right] } \\
& +\varepsilon_{n} c_{1 r}\left[f_{1}(\eta)+\ldots\right]+\ldots \\
c\left(\eta ; \varepsilon_{n}, \psi\right)= & {\left[c_{0}(\eta)+\psi c_{01}(\eta)+\ldots\right] } \\
& +\varepsilon_{n} c_{1 r}\left[c_{1}(\eta)+\ldots\right]+\ldots \\
H\left(\eta ; \varepsilon_{n}, \psi\right)= & {\left[H_{0}(\eta)+\psi H_{01}(\eta)+\ldots\right] } \\
& +\varepsilon_{n} c_{1 r}\left[H_{1}(\eta)+\ldots\right]+\ldots
\end{aligned}
$$

where $c_{1 r}$ is a parameter which will be determined subsequently in the matching of the inner and outer solutions.

Substitution of the expansions given by Eqs. (24) into Eqs. (1)-(3) and collection of powers in $\varepsilon_{n}$ and $\psi$ yield three sets of equations. The first given by the zero-order in each expansion is

$$
\begin{aligned}
f_{0}{ }^{\prime \prime \prime}+f_{0} f_{0}{ }^{\prime \prime}+\frac{1}{2}\left[1+\tau\left(H_{0}+c_{0}\right)-f_{0}{ }^{\prime 2}\right] & =0 \\
c^{\prime}{ }^{\prime \prime}+\sigma f_{0} c_{0}{ }^{\prime} & =0, \quad \eta>\eta_{r} \\
c_{0} & =1, \quad \eta<\eta_{r} \\
H_{0}{ }^{\prime \prime}+\sigma f_{0} H_{0}{ }^{\prime} & =0
\end{aligned}
$$

which are to be solved subject to the following boundary conditions:

$$
\begin{aligned}
f_{0}(0) & =0 \\
f_{0}(\infty) & =1, \quad c_{0}(\infty)=H_{0}(\infty)=0 \\
f_{0}{ }^{\prime}(-\infty) & =\left[1+\tau\left(H_{\infty}+1\right)\right]^{1 / 2}, \quad H_{0}(-\infty)=H_{\infty}
\end{aligned}
$$

The second set of equations relates to the first-order functions in $\varepsilon_{n}$ and is:

$$
\begin{aligned}
f_{1}^{\prime \prime \prime}+f_{0} f_{1}{ }^{\prime \prime}+f_{0}{ }^{\prime \prime} f_{1}+\frac{1}{2}\left[\tau\left(H_{1}+c_{1}\right)-2 f_{0}{ }^{\prime} f_{1}^{\prime}\right] & =0 \\
c_{1}{ }^{\prime \prime}+\sigma\left(f_{0} c_{1}{ }^{\prime}+c_{0}{ }^{\prime} f_{1}\right) & =0 \\
H_{1}{ }^{\prime \prime}+\sigma\left(f_{0} H_{1}{ }^{\prime}+H_{0}{ }^{\prime} f_{1}\right) & =0
\end{aligned}
$$

with boundary conditions

$$
\begin{aligned}
f_{1}(0) & =0 \\
c_{1}\left(\eta_{r}\right) & =1 \\
f_{1}^{\prime}(\infty) & =c_{1}(\infty)=H_{1}(\infty)=0 \\
f_{1}{ }^{\prime}(-\infty) & =c_{1}(-\infty)=H_{1}(-\infty)=0
\end{aligned}
$$

The third set of equations arises from the first-order terms in $\psi$ and is 


$$
\begin{aligned}
f_{01}{ }^{\prime \prime}+f_{0} f_{01}{ }^{\prime \prime}+f_{0}^{\prime \prime} f_{01}+\frac{1}{2}\left[\tau\left(H_{01}+c_{01}\right)-2 f_{0}{ }^{\prime} f_{01}{ }^{\prime}\right] & =0 \\
c_{01}{ }^{\prime \prime}+\sigma\left(f_{0} c_{01}{ }^{\prime}+c_{0}{ }^{\prime} f_{01}\right) & =\sigma f_{0} c_{0}{ }^{\prime}, \quad \eta>\eta_{r} \\
& =0, \quad \eta<\eta_{r} \\
H_{01}{ }^{\prime \prime}+\sigma\left(f_{0} H_{01}{ }^{\prime}+H_{0} f_{01}\right) & =c_{0}^{\prime \prime}, \quad \eta>\eta_{r} \\
& =0, \quad \eta<\eta_{r}
\end{aligned}
$$

with boundary conditions

$$
\begin{aligned}
f_{01} & =0 \\
f_{01}{ }^{\prime}(\infty) & =c_{01}(\infty)=H_{01}(\infty)=0 \\
f_{01}{ }^{\prime}(-\infty) & =c_{01}(-\infty)=H_{01}(-\infty)=0 \\
H_{01}\left(\eta_{r}{ }^{-}\right) & =H_{01}\left(\eta_{r}{ }^{+}\right) \\
H_{01}{ }^{\prime}\left(\eta_{r}+\right) & =H_{01}{ }^{\prime}\left(\eta_{r}{ }^{-}\right)+c_{0 r^{\prime}}
\end{aligned}
$$

where we retain the notation of the previous section and let $c_{0 r}{ }^{\prime} \equiv c_{0}{ }^{\prime}\left(\eta_{r^{+}}{ }^{+}\right)$but note that the subscript $a$ no longer implies adiabatic flow.

The first two sets of equations are those solved in LWII and yield the outer solutions for nonadiabatic flamelets with unity Lewis number while the third set describes the first order effect of nonunity values of that number. Numerical solutions involve the parameters $\sigma, \tau, H_{\infty}$ and $\eta_{r}$ and yield the following quantities of interest for subsequent developments: $c_{0 r}{ }^{\prime}, c_{01 r}, H_{0 r}, H_{0 r^{\prime}}{ }^{\prime} H_{01 r}$ and $H_{1 r}$. We give later representative results obtained from solution of the third set of equations to complement those given in LWII.

\subsection{The Inner Solutions}

The appropriate independent variable for resolution of the structure of the reaction zone is given in LWII, namely

$$
\xi=\left[\frac{\eta-\eta_{r}}{\varepsilon_{n}}\right] \frac{1}{a}+\beta
$$

where $a \equiv\left(h_{0 r}+\tau\right) /\left(-c_{0 r}{ }^{\prime}\right)$ and where $\beta$ is a translational factor to be determined later by matching the inner and outer solutions.

To describe the chemical term as $\varepsilon_{n} \rightarrow 0$ we need approxinate descriptions of the outer solutions for the enthalpy function and of the progress variable in the neighborhood of $\eta=\eta_{r}$; thus we have

$$
\begin{aligned}
& H\left(\eta ; \varepsilon_{n}, \psi\right) \approx H_{0 r}+\varepsilon_{n}\left[\chi_{L n} H_{01 r}+H_{0 r^{\prime}} \alpha(\xi-\beta)+c_{1 r} H_{1 r}\right]+\ldots \\
& c\left(\eta ; \varepsilon_{n}, \psi\right) \approx 1-\varepsilon_{n}\left(h_{0 r}+\tau\right)\left[y_{1}(\xi)+\ldots\right]
\end{aligned}
$$

where $X_{L n} \equiv \psi / \varepsilon_{n}=O(1)$ is a parameter analogous to $X$ and $\chi_{L}$ of the previous section. Thus as $\varepsilon_{n} \rightarrow 0$ the Lewis number approaches unity so that $\chi_{L n}$ remains $O(1)$. 
Substitution of all requisite quantities into Eq. (2) and retention of only the lowest order terms in $\varepsilon_{n}$ leads to

$$
y_{1}^{\prime \prime}=\left\{\Delta_{n} \frac{\left(h_{0 r}+\tau\right)^{3}}{\left(-c_{0 r}\right)^{2}} \exp \left[\frac{c_{1 r} H_{1 r}+\chi_{L n} H_{01 r}}{h_{0 r}+\tau}-\gamma \beta\right]\right\} y_{1} \exp \left[-\left(y_{1}-\gamma \xi\right)\right]
$$

where the enthalpy parameter $h_{0 r}$ has been set equal to $1+\tau H_{0 r}$ and may therefore be considered determined. The new quantity $\gamma \equiv H_{0 r}{ }^{\prime} /\left(-c_{0 r}{ }^{\prime}\right)$ is the so-called temperature gradient parameter and is determined by the outer solutions. The left side of Eq. (30) arises from the diffusion term of Eq. (2) while the right is from the chemical term. We thus have the usual diffusive-reactive balance operative in thin reaction zones. The solutions to Eq. (30) are required for the purposes of matching to satisfy the boundary conditions $y_{1}^{\prime}(\xi \rightarrow-\infty)=0, y_{1}^{\prime}(\xi \rightarrow \infty)=1$.

As noted in LWII Liñán (1974) studies Eq. (30) as it arises in nonpremixed flames and shows that the factor within the brackets on the right side equals $\frac{1}{2}$. Since matching determines $\beta$ and $c_{1 r}$ in terms of $\gamma$, we thus can determine the desired relation between $\Delta_{n}$ and the reaction zone location given by $\eta_{r}$. Liñan also shows that as $\xi \rightarrow \infty \quad y_{1} \rightarrow \xi+y_{10}$, that as $\xi \rightarrow-\infty y_{1} \rightarrow y_{1 \infty}$, that for $\gamma \leq 0 . y_{1 \infty} \equiv 0$ and finally that there are no solutions to Eq. (30) for $\gamma>\frac{1}{2}$. This last finding implies that for aerothermochemical conditions including the rate of strain resulting in $\gamma>\frac{1}{2}$ a thin reaction zone description of flamelet behavior is inapplicable. As in LWII we shall see later that under such conditions a diffuse reaction zone located far into the product stream prevails.

\subsection{The Matching Conditions}

To complete the calculations in this section we match the inner and outer solutions For $\xi \rightarrow \infty$ we have

$$
\lim _{\xi \rightarrow \infty}\left\{\left[c_{0 r^{\prime}} \alpha(\xi-\beta)+\chi_{L n} c_{01 r}+c_{1 r}\right]+\left(h_{0 r}+\tau\right)\left(\xi+y_{10}\right)\right\}=0
$$

By virtue of the definition of a the quantities multiplying the $\xi$-terms cancel and we are left with

$$
\left(-c_{0 r}\right)^{\prime} \alpha \beta+\chi_{L n} c_{01}+c_{1 r}=-\left(h_{0 r}+r\right) y_{10}
$$

Similarly, for $\xi \rightarrow-\infty$ we obtain

$$
\chi_{L n} c_{01 r}+c_{1 r}=-\left(h_{0 r}+\tau\right) y_{1 \infty}
$$

Equations (32) and (33) reduce as they should to the corresponding equations in LWII when the Lewis number is unity, i.e., when $X_{L n}=0$.

Thus we find

$$
\begin{aligned}
-c_{1 r} & =\left(h_{0 r}+\tau\right) y_{1 \infty}+\chi_{L n} c_{01 r} \\
\beta & =y_{1 \infty}-y_{10}
\end{aligned}
$$


We see that for $\gamma \geq 0$ so that $y_{1 \infty}=0 c_{1 r} \neq 0$ and the second-order solutions play a role in describing the effects of nonunity Lewis numbers.

We are now able to determine $\Delta_{n}$; with the factor within brackets on the right side of Eq. (30) set equal to $\frac{1}{2}$ and with $c_{1}$ and $\beta$ eliminated by means of Eqs. (34) we have

$$
\Delta_{n}=\frac{\left(-c_{0 r}{ }^{\prime}\right)^{2}}{2\left(h_{0 r}+\tau\right)^{3}} \exp \left[\chi_{L n}\left(\frac{c_{01 r} H_{1 r}-H_{01 r}}{h_{0 r}+\tau}\right)+\left(\gamma+H_{1 r}\right) y_{100}-\gamma y_{10}\right]
$$

which reduces properly to the corresponding equation in LWII if $\chi_{L n}=0$.

Equation (35) completes the sequence of calculations outlined earlier; for a specified set of thermochemical parameters and a specified value of $\eta_{r}$ all quantities on the right side of Eq. (35) including $\gamma$ are given by the outer solutions. The results in Liñan (1974) and/or numerical solutions of Eq. (30) determine $y_{10}$ and $y_{1 \infty}$ so that $\Delta_{n}$ and subsequently the rate of strain can be calculated.

The behavior of the fundamental Damköhler parameter $\Delta$ with reaction zone location for a given set of thermochemical conditions is given by combining Eqs. (35) and (23) and by specifying a value of $\varepsilon$. Clearly, the resulting expression $\Delta=\Delta\left(\eta_{r} ; \tau, H_{\infty}\right.$, $\left.\chi_{L}, \varepsilon\right)$ is too complex and contains too many implicit functions to permit quantitative conclusions to be drawn concerning such behavior. We must resort to the restricted but simpler analysis of the previous section for such purposes. In particular the conditions leading to extrema in $\Delta$ and thus to the possibility of flamelet extinction and ignition cannot be determined analytically. However, we know from the analysis and discussion in LWII of the connection between the two Damköhler parameters that generally qualitative information on the extrema in $\Delta$ is given by extrema in $\Delta_{n}$, qualitative because the extrema in the former correspond to different values of $\eta_{r}$ and to significantly different values of the two Damköhler parameters [cf. Eq. (23)]. The only ambiguity in this information relates to flows involving slightly cooled product streams, i.e., to $H_{\infty} \rightarrow 0^{-}$, wherein monotonic variations in $\Delta_{n}$ can result in nonmonotonic variations and therefore extrema in $\Delta$.

Despite the clutter connected with Eq. (35) and the difficulty in assessing flamelet behavior that results it is possible to identify a crucial parameter describing the influence of nonunity Lewis numbers. From the combination of Eqs. (35) and (23) we can write

$$
\Delta=\Delta_{L e=1} \exp \left(-\chi_{L} \Lambda\right)
$$

where

$$
\Lambda \equiv-\frac{\left(1+{ }_{r}\right)\left(c_{01 r} H_{1 r}-H_{01 r}\right)}{\left(h_{0 r}+\tau\right)^{2}}=\Lambda\left(\eta_{r} ; \tau, H_{\infty}\right)
$$

If Eq. (36) is compared with its counterpart for nearly adiabatic flamelets, namely with Eq. (17), we see that $\Lambda=H_{\psi r} /(1+\tau)$ if $H_{\infty} \approx 0$. By examining the characteristics of $\Lambda$ we shall be able to determine the effect of nonunity Lewis numbers on $\Delta$ for general, nonadiabatic flamelets. Moreover, we know from LWI and LWII that the parameter describing the rate of creation of product $m$ for the present case is

$$
m=\frac{\left(-c_{0 r}^{\prime}\right)^{2}}{(2 \Delta)^{1 / 2}(1+\tau)^{3 / 2}}
$$


Thus the parameter $\Lambda$ can also be used to assess the influence of nonunity Lewis numbers on the rate of creation of product via its effect on $\Delta$. Of course, these effects for nearly adiabatic flamelets are given by the analysis of the previous section and for for the specific case of $t=3$ by Figures 10 and 11 .

\subsection{Results for Nonadiabatic Flamelets with Lewis Numbers Near Unity}

The analysis of this section involves a large number of parameters each with a wide range of possible values. Accordingly, as in LWII we must restrict our calculations to representative and especially illuminating values thereof. Thus most of our results pertain to a value of the heat release parameter $\tau=3$ while we choose a range of values of $h_{\infty}=\left(H_{\infty}-1\right) / \tau$ from one corresponding to a superadiabatic product stream, $h_{\infty}=1.5$, to a highly cooled stream, $h_{\infty}=-1$. Finally, we note that when necessary to display the results in terms of parameters of direct physical significance, we chose a value of $\varepsilon=0.02$ which corresponds for $\tau=3$ to $T_{a}=67$, a realistic activation temperature [cf. Eqs. (5)]. In the same spirit we present results for specific values of $\chi_{L}=$ $\chi_{L n}\left(\varepsilon_{n} / \varepsilon\right)$ rather than of $\chi_{L n}$ corresponding to values of the Lewis number greater than and less than unity.

For the case considered in this section the outer solutions to the equations for the zero- and first-orders in $\varepsilon_{n}$ are given in LWII for a range of parameters $\tau, h_{\infty}$ and $\eta_{r}$ and need not be repeated here. The solutions corresponding to the first-order perturbations in $\psi$ are new; however, in the interest of brevity we present only the results of greatest physical significance and show in Figure 12 the distributions of the per-

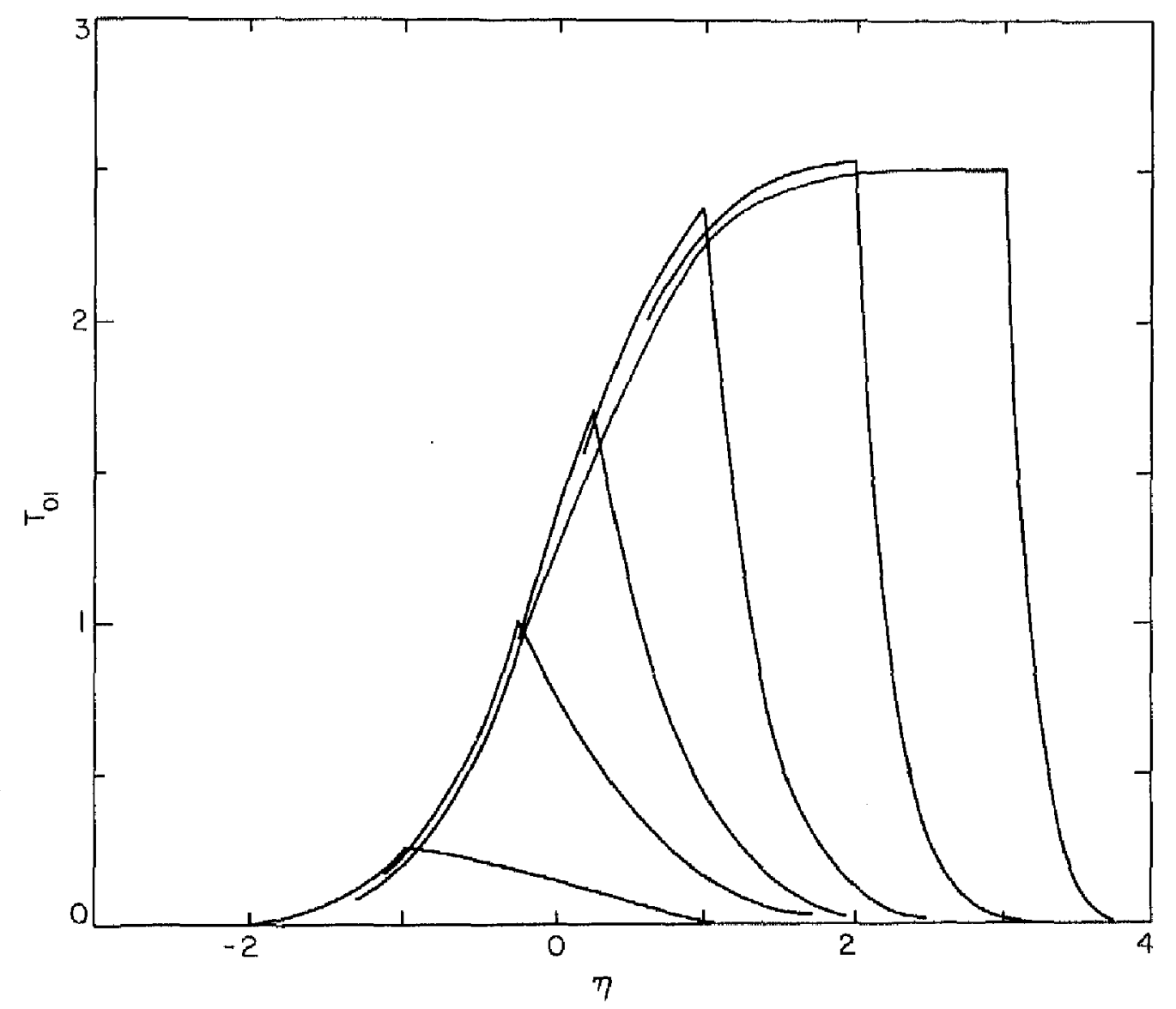

FIGURE 12 The perturbed temperature profiles associated with $L e \neq 1: \tau=3$, his $=1.5$. 
turbed temperature $T_{01}(\eta)$ for various reaction zone locations and for $h_{\infty}=1.5$ and $\tau=3$. The distributions for other values of $h_{\infty}$ and $\tau$ are not significantly different. We see that the distributions of $H_{01}(\eta)$ such as suggested by Figure 2 and of $c_{01}(\eta)$ lead to $T_{01}(\eta)>0$. Thus the perturbation of the temperature within a flamelet is uniformly positive or negative depending on the sign of $\psi$; if $\psi>0(L e<1)$, the temperature is increased while for $\psi<0(L e>1)$, the temperature is reduced. The influence of such perturbations on the occurrence of abrupt transitions in reaction zone location and on the rate of creation of product is discussed in Section 3.5.

Our earlier discussion establishes that the parameter $\Lambda$ defined by Eq. (36) is crucial in determining the effect of nonunity Lewis numbers on several flamelet characteristics. Thus we show in Figure 13 the variation thereof, again for $\tau=3$. First, we note that for the adiabatic case, $h_{\infty}=1$, the distribution of $\Lambda$ is in excellent agreement with its

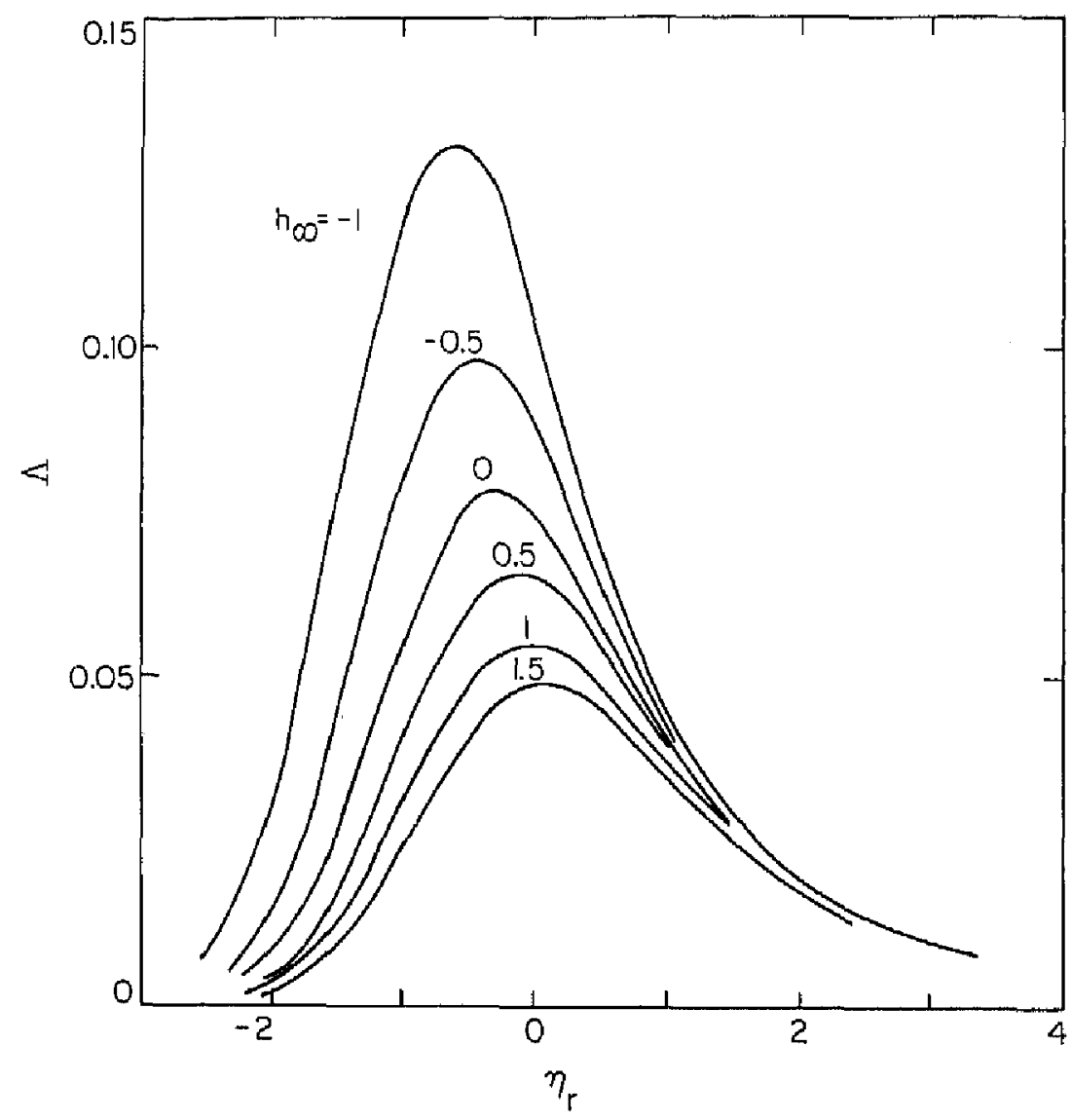

FIGURE 13 The variation of the Lewis number parameter $\Lambda$ with reaction zone location: $r=3$.

counterpart from the analysis of the previous section, i.e., with $H_{\psi 1 r} /(1+\tau)$. We see from Figure 13 that for $0.5<h_{\infty}<1.5$ which corresponds for $\tau=3$ to $\left|H_{\infty}\right|<0.167$ the nearly adiabatic solution provides a satisfactory measure of the influence of nonunity Lewis numbers on the Damköhler parameter. For greater degrees of product cooling the values of $\Lambda$ and thus flamelet sensitivity to nonunity Lewis numbers increase. Accordingly, our earlier conclusions regarding such sensitivity prevail over the entire range of enthalpies in the product stream: If the Lewis number is less than unity, 
$X_{L}>0, \Delta<\Delta_{L e=1}$ and a greater rate of strain corresponds to a given reaction zone location and a given rate of creation of product.

We next present the generalization of the results of Figures 6 and 10 in terms of the Damköhler parameter $\Delta_{n}$ versus reaction zone location $\eta_{r}$ in Figures 14a and 14b.

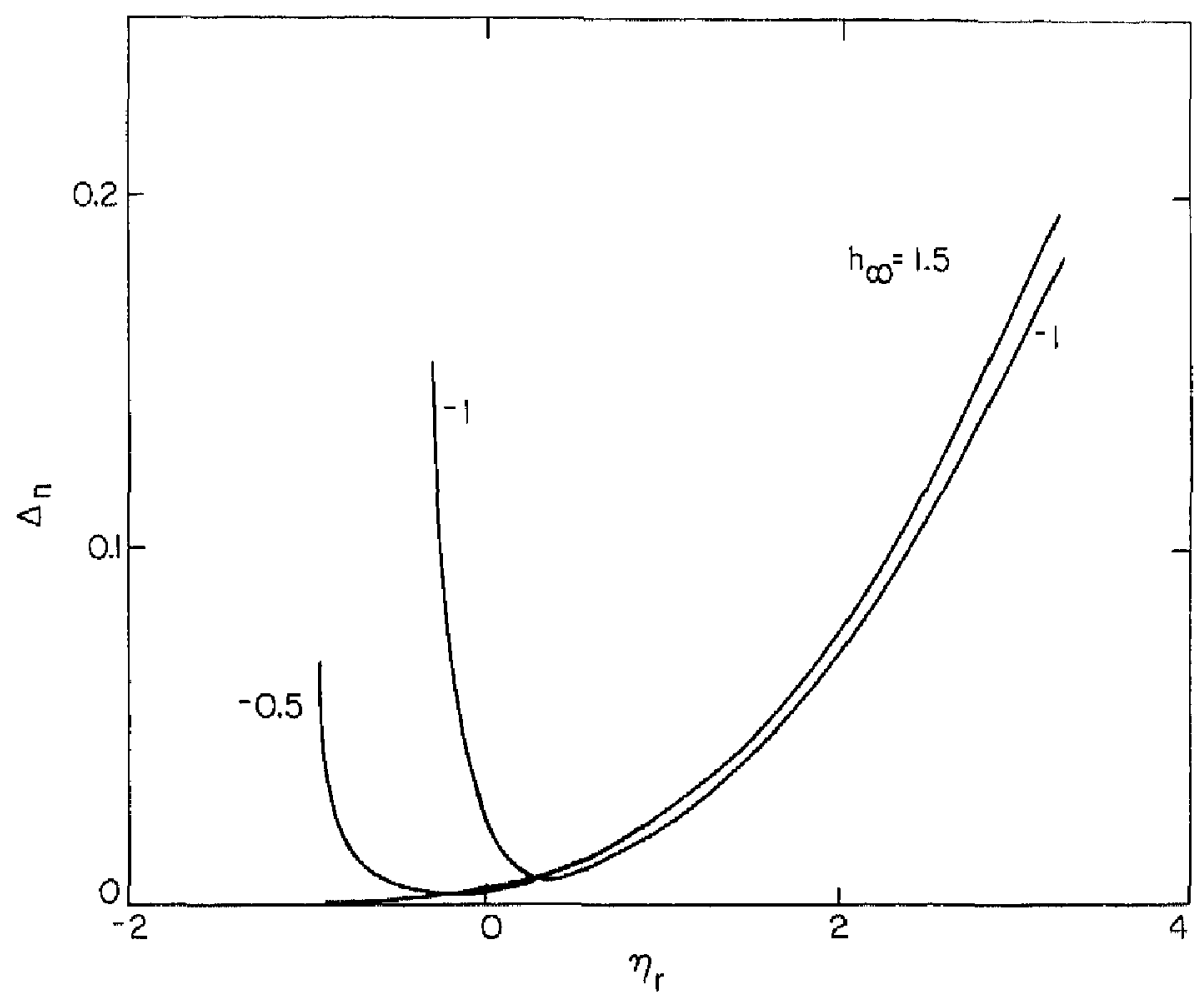

FIGURE 14 The variation of the Damköhler parameter $\Delta_{n}$ with reaction zone location: $\tau=3$. (a) $\chi_{L}=10$, (b) $x_{L}=-10$.

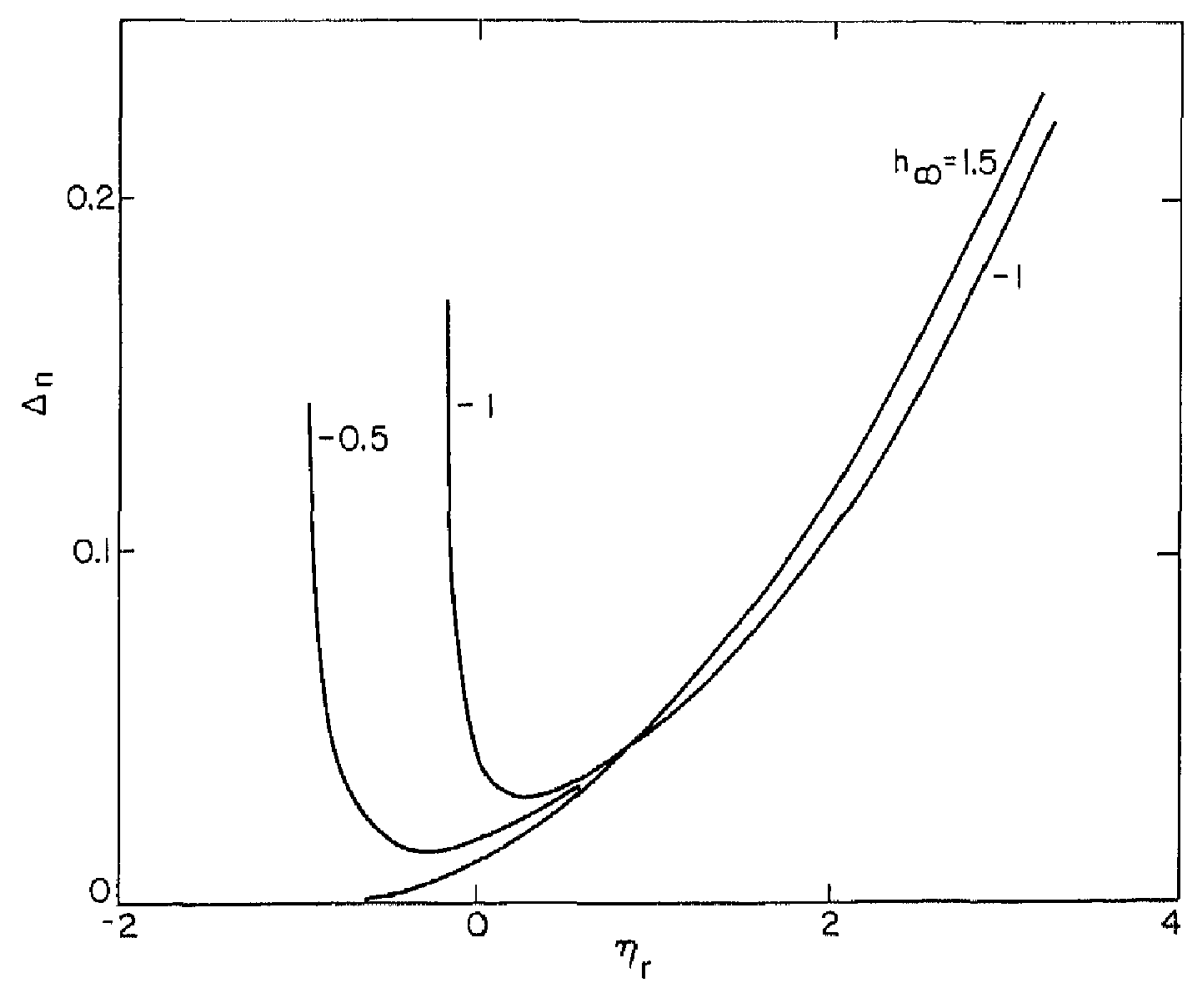


Two values of $X_{L}$ are considered; for the value $\varepsilon=0.02$ and for values $|\psi| \approx 0.2$ which may reasonably be assumed the maximum appropriate for an analysis of near unity Lewis numbers values of $X_{L}= \pm 10$ can be considered representative.

Both Figures $14 \mathrm{a}$ and $14 \mathrm{~b}$ show that in terms of $\Delta_{n}$ significant degrees of product cooling are required for extrema in $\Delta_{n}$ to prevail, a result consistent with findings in LWII. However, it is important to recall in this connection that monotonic variations

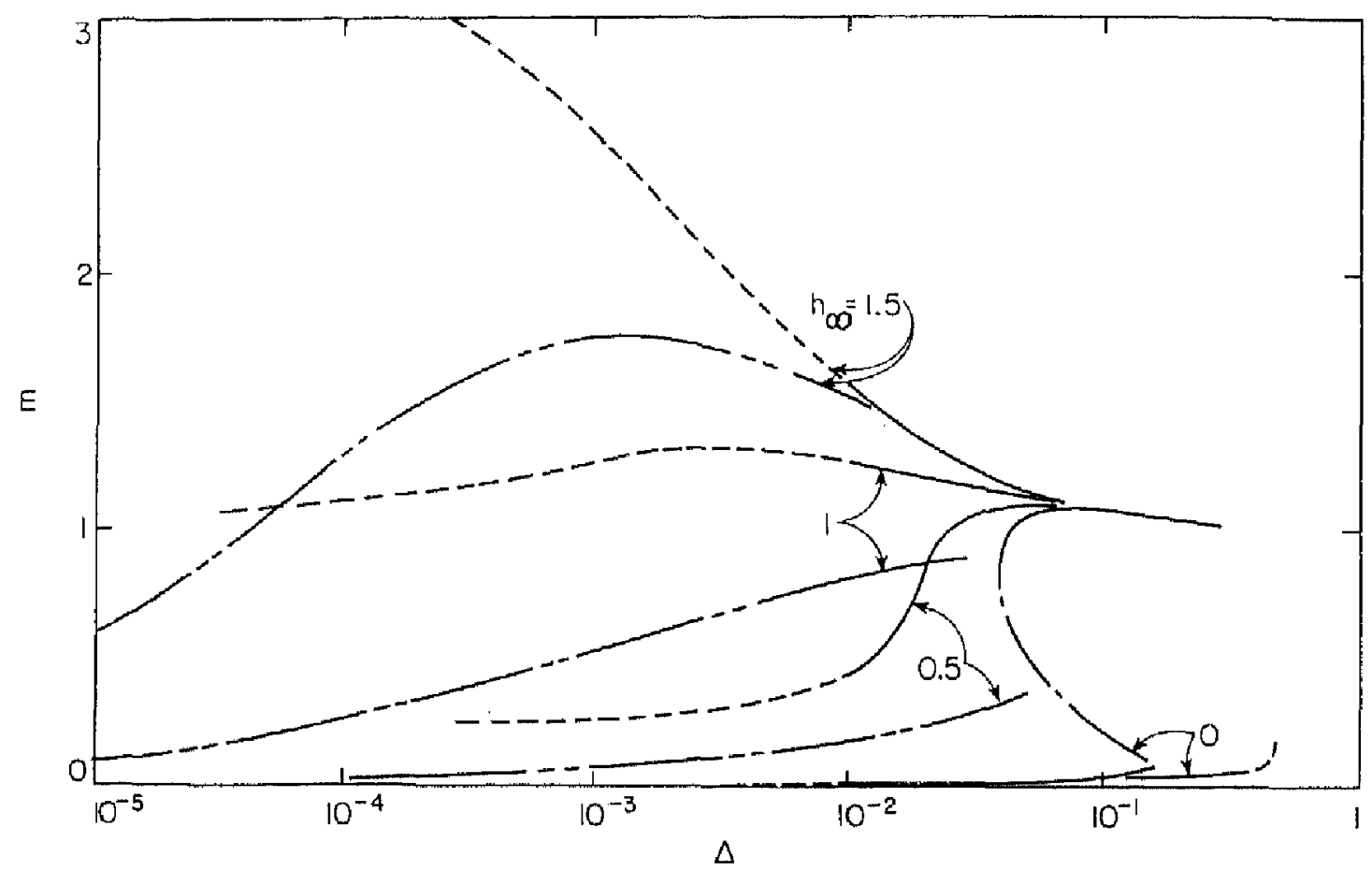

FIGURE 15 The variation of the mass rate of creation parameter with Damköhler parameter: $\tau=3$. (a) $x_{L}=10$, (b) $x_{L}=-10$.

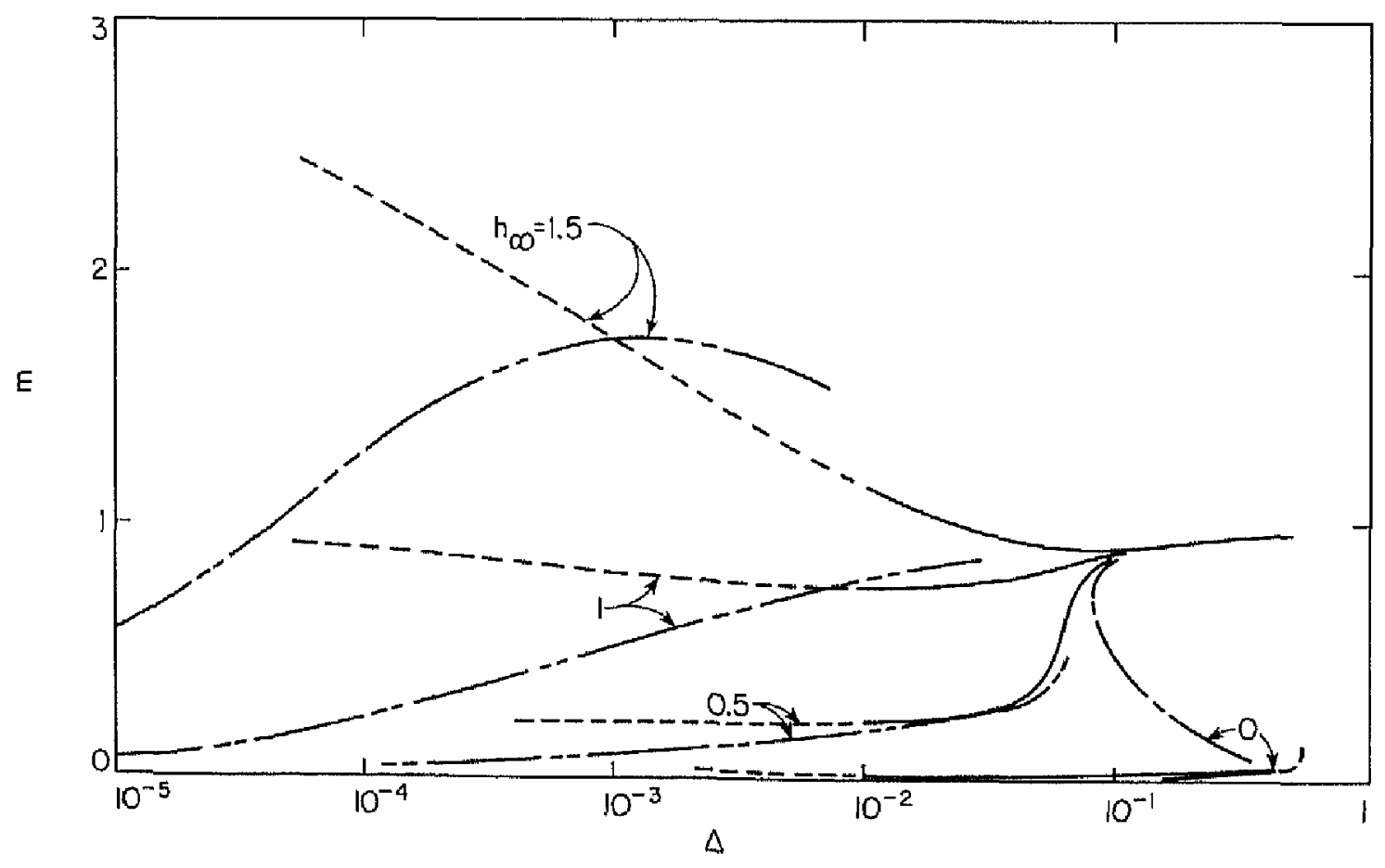


in $\Delta_{n}$ can result in nonmonotonic variations of $\Delta$ for subadiabatic flamelets. A comparison of these figures indicates that as in the previous section extrema are more likely to occur if $\chi_{L}<0$, i.e., if the Lewis number is greater than unity.

The results in Figures $14 \mathrm{a}$ and $14 \mathrm{~b}$ correspond to the variations with the Damköhler parameter $\Delta$ of the rate of product creation as indicated by the parameter $m$ given in Figures $15 \mathrm{a}$ and $15 \mathrm{~b}$. These figures represent extensions of Figures $11 \mathrm{a}$ and $11 \mathrm{~b}$ to arbitrary degrees of product cooling. The multiple values of $m$ for a given $\Delta$ correspond to extrema in $\Delta$ versus $\eta_{r}$. We see that for both values of $X_{L}$ a value of $h_{x} \approx 0.5$ separates flamelet behavior into continuous and discontinuous modes as the rate of strain is altered. Again we see that for Lewis numbers greater than unity the critical value of $h_{\infty}$ is greater, i.e., a flamelet with $L e>1$ can tolerate less product cooling before being susceptible to abrupt transitions in reaction zone location and in rate of product creation resembling extinction or ignition. Finally, we again note the significant influence of nonunity Lewis numbers on the parameter $m$ for low rates of strain; briefly, the approach of $m$ to unity differs for the two values of $\chi_{L}$.

\section{SOLUTIONS FOR HIGH RATES OF STRAIN}

As the rate of strain to which a flamelet is subjected increases such that $-\eta_{r} \rightarrow \infty$ the discontinuity at the reaction zone weakens, the thickness of that zone increases [cf. Eq. (28)] and our analysis in the previous section applicable to thin reaction zones no longer prevails. As $-\eta_{r} \rightarrow \infty$ the condition $c_{n}\left(\eta_{r}\right)=1$ applied to the outer solutions can be effectively replaced by $c_{0}(\eta \rightarrow-\infty)=1$. But solutions to Eqs. (1)-(3) with the right side of Eq. (2) set to zero and with this replacement describe a so-called frozen flow which forms the basis for an analysis of flamelets subjected to high rates of strain.

The analysis closely follows that given in LWII which should be consulted for details. Briefly, asymptotic approximations to the frozen flow solutions valid for $-\eta \rightarrow \infty$ represent the outer solutions in the neighborhood of an extended reaction zone and are used to identify a value of $\eta$ denoted $\eta^{*}$ within that zone. A new variable $\xi$ is introduced so that diffusive, convective and reactive effects are all retained in the inner solutions which are given by an equation involving in a quite natural way a new Damköhler parameter related in due course to $\Delta$. Thus comparison between the results of the analysis of this section which pertains to exceedingly small values of $\Delta$ can be made with those obtained earlier for $\Delta=O(1)$.

\subsection{The Frozen Flow Solutions}

Following LWII, we observe that the asymptotic behavior of the frozen fiow solutions as $-\eta \rightarrow \infty$ are

$$
\begin{aligned}
f_{f} & \approx\left[1+\tau\left(H_{\infty}+1\right)\right]^{1 / 2}\left(\eta+\kappa_{f}\right) \\
c_{f} & \approx 1+\frac{A_{f}}{\eta+\kappa_{f}} \exp \left[-\frac{\sigma L e}{2}\left[1+\tau\left(H_{\infty}+1\right)\right]^{1 / 2}\left(\eta+\kappa_{f}\right)^{2}\right] \\
\frac{T_{f}-1}{\tau} & =H_{f}+c_{f} \\
& \approx\left(1+H_{\infty}\right)\left\{1+\frac{B_{f}}{\eta+\kappa_{f}} \exp \left[-\frac{\sigma}{2}\left[1+\tau\left(H_{\infty}+1\right)\right]^{1 / 2}\left(\eta+\kappa_{f}\right)^{2}\right]\right\}
\end{aligned}
$$


where $A_{f}, \kappa_{f}$ and $B_{f}$ are parameters whose values are given by numerical solution of the equations for frozen flow. Without loss of clarity we henceforth drop the subscript on these parameters.

Several preliminary remarks are indicated. We are interested in flows with $T_{\infty}>1$, i.e., in flamelets having their highest temperature in the product stream, and we thus restrict $H_{\infty}>-1$. Furthermore, we modify Eqs. (22) appropriately by replacing $\Delta_{n}$ with $\Delta_{w}, \varepsilon_{n}$ with $\varepsilon_{\infty}$ and by setting $h_{0 r}=1+\tau H_{\infty}$. These replacements apply to Eqs. (23) as well so that we have relations for the fundamental parameters $\Delta$ and $\varepsilon$ in terms of the parameters $\Delta_{\infty}$ and $\varepsilon_{\infty}$. When we compare the results of Section 4 for $\Delta \ll 1$ with those of the present section as the high rates of strain decrease, we must specify a value for $\varepsilon$ and use Eqs. (23) with the cited replacements. These observations are also noted in LWII.

To proceed we identify a value of $\eta \equiv \eta^{*}$ within the extended reaction zone such that $T\left(\eta^{*}\right)=\left(T_{\infty}-\varepsilon_{\infty} \tau\right)$ since for $\eta>\eta^{*}$ chemical reaction is negligible and the frozen flow solutions prevail. From the last of Eqs. (38) we get

$$
\varepsilon_{\infty} \tau=-\tau\left(H_{\infty}+1\right) \frac{B}{\eta^{*}+\kappa} \exp \left[-\frac{\sigma}{2}\left[1+\tau\left(H_{\infty}+1\right)\right]^{1 / 2}\left(\eta^{*}+\kappa\right)^{2}\right]
$$

The derivative of $c_{f}$ is needed; from the second of Eqs. (38) we find

$$
c_{f}^{\prime} \approx-\sigma L e A\left[1+\tau\left(H_{\infty}+1\right)\right]^{1 / 2} \exp \left[-\frac{\sigma L e}{2}\left[1+r\left(H_{\infty}+1\right)\right]^{1 / 2}(\eta+\kappa)^{2}\right]
$$

\subsection{Solutions for the Reaction Zone}

The expansion of $c(\eta)$ needed to resolve the structure of the reaction zone is given by Eq. (29) with $\xi$ replaced by a new independent variable $\zeta$ and with $h_{0 r}=1+\tau\left(H_{\infty}+1\right)$, a determined value. As in LWII $\zeta$ is defined by

$$
\zeta=-\frac{A}{\varepsilon_{\infty}\left[1+\tau\left(H_{\infty}+1\right)\right](\eta+\kappa)} \exp \left[-\frac{\sigma L e}{2}\left[1+\tau\left(H_{\infty}+1\right)\right]^{1 / 2}(\eta+\kappa)^{2}\right]
$$

Thus the structure of the reaction zone is given by $y_{1}(\zeta)$.

At this juncture we restrict attention to values of the Lewis number near to unity and introduce once again the parameter $\psi \equiv L e^{-1}-1$. To lowest order in $\psi$ Eq. (3) rewritten with $\zeta$ as the independent variable yields $H^{\prime \prime}(\zeta) \approx 0$ so that $H \approx H_{\infty}+B_{h} \zeta$ where $B_{h}$ is an integration constant to be determined from the second and third of Eqs. (38) and from Eq. (41). To lowest order in $\psi$ we find

$$
\begin{gathered}
B_{h}=\varepsilon_{\infty}\left[1+\tau\left(H_{\infty}+1\right)\right] \\
\left\{1-\left(1+H_{\infty}\right) \frac{B}{A} \exp \left[-\frac{\sigma L e \psi}{2}\left[1+\tau\left(H_{\infty}+1\right)\right]^{1 / 2}\left(\eta^{*}+\kappa\right)^{2}\right]\right\}
\end{gathered}
$$

This equation is valid for $\psi\left(\eta^{*}+\kappa\right)^{2}$ of order unity or less, i.e., for $\psi$ of order $\left(-\ln \varepsilon_{\infty}\right)^{-1}$ or less. The quotient B/A requires numerical evaluation if $\psi$ is of this order but if $\psi$ 
is sufficiently small so that $\psi\left(\eta^{*}+\kappa\right)^{2} \ll 1$, then it can be shown that to a first approximation $B / A \approx 1$ and a simpler formula results, namely

$$
\begin{gathered}
B_{h} \approx \varepsilon_{\infty}\left[1+\tau\left(H_{\infty}+1\right)\right] \\
{\left[-H_{\infty}+\left(1+H_{\infty}\right) \frac{\sigma L e \psi}{2}\left[1+\tau\left(H_{\infty}+1\right)\right]^{1 / 2}\left(\eta^{*}+\kappa\right)^{2}\right]}
\end{gathered}
$$

With either Eq. (42) or Eq. (43) as appropriate we can rewrite the enthalpy in the form $H(\zeta)$ within the reaction zone as

$$
H \approx H_{\infty}+\varepsilon_{\infty} \lambda\left[1+\tau\left(H_{\infty}+1\right] \zeta\right.
$$

where the important parameter $\lambda$ is defined as

$$
\lambda \equiv-H_{\infty}+\frac{1}{2}\left(1+H_{\infty}\right) \sigma \psi\left[1+\tau\left(H_{\infty}+1\right)\right]^{1 / 2}\left(\eta^{*}+\kappa\right)^{2}
$$

when Eq. (43) applies. If $\psi=0$ we recover the definition of $\lambda$ in LWII. It is to be noted that only when Eq. (42) is needed will $\lambda+H_{\infty}$ be a quantity of order unity. When $\psi$ is of an order smaller than $\left(-\ln \varepsilon_{\infty}\right)^{-1}$, i.e., when $\psi$ is in the range of practical interest, then $\lambda=-H_{\infty}$ to lowest order in the expansion in $\varepsilon$. But this is the same value of $\lambda$ arising in LWII. Henceforth we restrict our attention to this case which corresponds to departures of the Lewis number from unity encountered in real gas mixtures. As a consequence the high-strain analysis of LWII applies here.

It is interesting that rather large departures of the Lewis number from unity are needed to influence flamelet behavior under conditions of high rates of strain. This observation is consistent with the previously noted relative insensitivity to deviations of the Lewis number from unity compared with deviations from adiabaticity found to occur when the reaction zone lies on the product side of the stagnation point.

When the various contributions are substituted in Eq. (2), we obtain the equation for $y_{1}(\zeta)$ to lowest order in $\psi$, namely

$$
\zeta^{2} y_{1}^{\prime \prime}=\delta y_{1} \exp \left[-\left(y_{1}-\lambda \zeta\right)\right]
$$

where $\delta$ is a new Damköhler parameter defined as

$$
\delta=\frac{\Delta_{\infty}}{\sigma^{2} \varepsilon_{\infty}^{2}\left(\eta^{*}+\kappa\right)^{2}} \approx \frac{\Delta_{\infty}\left[1+\tau\left(H_{\infty}+1\right)\right]^{1 / 2}}{2 \sigma \varepsilon_{\infty}^{2}\left(-\ln \varepsilon_{\infty}\right)}
$$

Equation (45) with its accompanying definition of $\delta$ is identical with the corresponding equation in LWII, although the extended definition of $\lambda$ could contain the influence of deviations of Lewis number from unity. It is an equation also studied by Liñan and Crespo (1976) in connection with nonpremixed laminar flames so that its character is understood. Briefly, $y_{1}(\zeta \rightarrow \infty) \rightarrow\left(\zeta-\phi_{\infty}\right)$ where $\phi_{\infty}$ depends on $\lambda$ and $\delta$ while matching with the frozen flow requires $y_{1}(0)=0$.

It is worth noting that the replacement of $\left(\eta^{*}+\kappa\right)$ in the second equality defining $\delta$ involves an approximation arising from Eq. (39), namely that $\left(\eta^{*}+\kappa\right)^{2} \gg 1$. In LWII it is found that with values of $\varepsilon$ of applied interest this inequality is only marginally respected and that the use of Eq. (39) without further approximation is required. 
Nevertheless the approximate determination of $\delta$ is instructive since it clearly indicates that for $\delta=O(1)$ and for $\varepsilon_{\infty} \ll 1$ the corresponding values of $\Delta_{\infty}$ are exceedingly small.

Formulas for the rate of creation of product as reflected in the parameter $m$ in the high-strain case are the same as those given in LWII. Thus it is found that

$$
m=\left[\frac{1+\tau\left(H_{\infty}+1\right)}{1+\tau}\right]^{5 / 2}-\frac{\phi_{\infty}}{\operatorname{Le}(2 \delta)^{1 / 2}} \exp \left[\frac{H_{\infty}}{2 \varepsilon\left[1+\tau\left(H_{\infty}+1\right)\right]}\right]
$$

where $\phi_{\infty}$ is given as a function of $\delta$ for various values of $\lambda$ in LWII.

Equation (46) is used to add to Figures $15 \mathrm{a}$, b the results for high rates of strain, i.e., for small $\Delta$. The agreement in Figures $15 \mathrm{a}, \mathrm{b}$ between the results for high and moderate rates of strain is comparable to that found in LWII for $X_{L}=0$. The reasons for the mismatch and criteria for deciding whether to use the high-strain or moderate-strain results are discussed in LWII.

\section{CONCLUSIONS}

This paper completes our investigations of the influence of strain on premixed laminar fla mes using the method of activation energy asymptotics by extending previous studies to include the influence of Lewis numbers different from unity. As expected from earlier work such as that of Sivashinisky (1976) it is found that Lewis numbers less than unity lead to increases in the temperature at the reaction zone and in the rate of creation of products while Lewis numbers greater than unity lead to decreases in these quantities. Under adiabatic conditions this effect is found to result in abrupt transitions associated with ignition and extinction for Lewis numbers greater than unity provided the rate of strain is such that the reaction zone is on the reactant side of the stagnation point. Unexpectedly, the same transitions are found to occur for Lewis numbers less than unity when the reaction zone is on the product side of the stagnation point. A physical explanation of this new phenomenon is given. It is found, however, that in laminar flames with degrees of heat release of practical interest rather large deviations of the Lewis number from unity are needed if abrupt transitions are to occur in adiabatic flows and that therefore in practical situations abrupt extinction of stagnation point flames are unlikely to be observed as a consequence of the effects of Lewis numbers alone. Alternatively, the effects of nonadiabaticity appear to be of greater importance in causing abrupt transitions associated with extinction and ignition. A reexamination of the interpretation of experimental results such as those of Tsuji and Yamaoka (1981) therefore appears to be called for with account taken of the influence of variable density associated with heat release.

Another significant finding of the present study is that influences of nonunity Lewis numbers are large compared with those of nonadiabaticity when the rate of strain is suitably small so that the reaction zone is located far into the reactant stream. On the contrary the influences of nonadiabaticity dominate when the rate of strain is large and the reaction zone is far into the product stream. This finding which is explicable on aerothermochemical grounds establishes the ranges of the rates of strain within which it is most important to consider the effects of Lewis number and nonadiabaticity. Stated alternatively at low rates of strain nonadiabaticity may be ignored while at the high rates of strain for which the reaction zone involves a diffusive-convective-reactive 
balance nonunity Lewis numbers result in a higher-order effect that may be ignored to a first approximation.

Density variations associated with realistic values of the heat release are emphasized. The tendency for such variations to insulate the reaction zone from the external streams is found to be responsible for a decreasing sensitivity of flame behavior with increasing heat release. The numerical examples are chosen to exhibit the effects of degrees of heat release of practical interest in both turbulent flames and in laboratory experiments.

There are a number of other problems involving strained premixed flames with density variations taken into account calling for attention. These include the back-toback reaction zones of Tsuji and Yamaoka (1981) and the flame-tip of conical Bunsen flames. In addition further studies are required to clarify the influence of density changes, nonadiabaticity and nonunity Lewis numbers on premixed flames with chemical reactions treated in a one-step approximation. Finally, it would be of interest to study the influences of multiple-step reactions by the method of activation energy asymptotics for the flamelets treated in this series.

\section{Appendix}

\section{CALCULATION OF THE DERIVATIVE WITH RESPECT TO REACTION ZONE LOCATION}

For the determination of the conditions leading to extrema in nearly adiabatic flamelets with Lewis numbers near unity via Eq. (18) we must calculate various derivatives with respect to $\eta_{r}$. To do so the expansions of Eq. (6) must be extended to include a perturbation of $\eta_{r}$. The proper perspective calls for consideration of all dependent variables to be dependent on both $\eta$ and $\eta_{r}$. If we restrict attention to the lowest order terms in all three expansion parameters, it is useful to write

$$
\begin{aligned}
f\left(\eta ; \eta_{r}\right)= & f_{0}\left(\eta ; \eta_{r 0}\right)+\frac{\partial f_{0}}{\partial \eta_{r}}\left(\eta ; \eta_{r 0}\right)\left(\eta_{r}-\eta_{r 0}\right)+\ldots \\
c\left(\eta ; \eta_{r}\right)= & c_{0}\left(\eta ; \eta_{r 0}\right)+\frac{\partial c_{0}}{\partial \eta_{r}}\left(\eta ; \eta_{r 0}\right)\left(\eta_{r}-\eta_{r 0}\right)+\ldots \\
H\left(\eta ; \eta_{r}, H_{\infty}, \psi\right)= & H_{\infty}\left[H_{H 1}\left(\eta ; \eta_{r 0}\right)+\frac{\partial H_{H 1}}{\partial \eta_{r}}\left(\eta ; \eta_{r 0}\right)\left(\eta_{r}-\eta_{r 0}\right)+\ldots\right] \\
& +\psi\left[H_{\psi 1}\left(\eta ; \eta_{r 0}\right)+\frac{\partial H_{\psi 1}}{\partial \eta_{r}}\left(\eta ; \eta_{r 0}\right)\left(\eta_{r}-\eta_{r 0}\right)+\ldots\right]+\ldots
\end{aligned}
$$

where $\eta_{r 0}$ is a nominal value of $\eta_{r}$. Now we apply Eqs. (A-1) at $\eta_{r}=\eta_{r 0}+\delta, \delta \ll 1$ and rewrite them in the convenient form 


$$
\begin{aligned}
f\left(\eta ; \eta_{r 0}+\delta\right) & =f_{0}\left(\eta ; \eta_{r 0}\right)+c_{0 r}^{\prime} \hat{f}\left(\eta ; \eta_{r 0}\right) \delta+\ldots \\
c\left(\eta ; \eta_{r 0}+\delta\right)= & c_{0}\left(\eta ; \eta_{r 0}\right)+c^{\prime}{ }_{0 r} \hat{c}\left(\eta ; \eta_{r 0}\right) \delta+\ldots \\
H\left(\eta ; \eta_{r 0}+\delta, H_{\infty}, \psi\right)= & H_{\infty}\left[H_{H 1}\left(\eta ; \eta_{r 0}\right)+c^{\prime} \hat{H}_{1}\left(\eta ; \eta_{r 0}\right) \delta+\ldots\right] \\
& +\psi\left[H_{\psi 1}\left(\eta ; \eta_{r 0}\right)+c_{0 r}^{\prime} \hat{H}_{2}\left(\eta ; \eta_{r 0}\right) \delta+\ldots\right]+\ldots
\end{aligned}
$$

where $c_{0 r}^{\prime}=c_{0}^{\prime}\left(\eta=\eta_{r}+0 ; \eta_{r 0}\right)$ is the parameter introduced earlier and where as throughout the analysis ( $)^{\prime}$ denotes differentiation with respect to $\eta$.

The equations for $\hat{f}$ and $\hat{c}$ are found by substitution into Eqs. (1)-(2) and collection in powers of $\delta$ alone. There result

$$
\begin{aligned}
\hat{f}^{\prime \prime \prime}+f_{0} \hat{f}^{\prime \prime}+f^{\prime \prime}{ }_{0} \hat{f}+\frac{1}{2}\left(\tau \hat{c}-2 f^{\prime}{ }_{0} \hat{f}^{\prime}\right) & =0 \\
\hat{c}^{\prime \prime}+\sigma\left(f_{0} \hat{c}^{\prime}+\hat{c}^{\prime}{ }_{0} \hat{f}\right) & =0, \quad \eta>\eta_{r 0} \\
\hat{c} & =0, \quad \eta<\eta_{r 0}
\end{aligned}
$$

which are to be solved subject to obvious homogeneous boundary conditions at $\pm \infty$.

The equations for $\hat{H}_{1}$ and $\hat{H}_{2}$ are obtained by substitution into Eq. (3) and by collecting successively terms in $\delta H_{\infty}$ and $\delta \psi$. We obtain

$$
\begin{aligned}
& \hat{H}_{1}{ }^{\prime \prime}+\sigma\left(f_{0} \hat{H}_{1}^{\prime}+H_{H 1}^{\prime} \hat{f}\right)=0 \\
& \hat{H}^{\prime \prime}{ }_{2}+\sigma\left(f_{0} \hat{H}_{2}^{\prime}+H^{\prime}{ }_{\psi 1} \hat{f}\right)=\hat{c}^{\prime \prime}
\end{aligned}
$$

which are also subject to homogeneous conditions at $\pm \infty$ but to forcing conditions at $\eta=\eta_{r 0}$.

We considered these latter conditions; from the second of Eqs. (A-2) we have

$$
c\left(\eta=\eta_{r 0}+\delta ; \eta_{r 0}+\delta\right)=1 \approx 1+c^{\prime}{ }_{0 r} \delta+c^{\prime} \hat{0} \hat{c}\left(\eta=\eta_{r 0} ; \eta_{r 0}\right) \delta+\ldots
$$

or

$$
\hat{\imath}\left(\eta=\eta_{r 0} ; \eta_{r 0}\right)=-1
$$

a result which justifies the form taken for Eqs. (A-2). The solutions we seek are forced by this condition alone.

A simitar calculation leads to

$$
c^{\prime}\left(\eta=\eta_{r 0}+\delta ; \eta_{r 0}+\delta\right) \approx c_{0 r}^{\prime}-\sigma f_{0 r} c_{0}^{\prime} \delta+c_{0 r}^{\prime} \hat{c}^{\prime}\left(\eta=\eta_{r 0}+; \eta_{r 0}\right) \delta+\ldots
$$

with the consequence that one of the desired derivatives is

$$
\frac{\partial c^{\prime} 0 r}{\partial \eta_{r}}=-o f_{0 r} c^{\prime}{ }_{0 r}+c^{\prime}{ }_{0 r} \hat{c}^{\prime}\left(\eta=\eta_{r 0}{ }^{+} ; \eta_{r 0}\right)
$$


An exactly similar calculation without difficulty leads to

$$
\frac{\partial H_{H 1 r}}{\partial \eta_{r}}=H_{H 1 r}^{\prime}+c^{\prime}{ }_{0 r} \hat{M}_{1}\left(\eta=\eta_{r 0} ; \eta_{r 0}\right)
$$

The situation with respect to the final derivative, that related to $H_{\psi t 1 r}$, is somewhat more complicated as a consequence of the discontinuity in the derivative of $H_{\psi 1}$ at the reaction zone. We calculate first the condition on $\hat{H}_{2}$ at $\eta=\eta_{r 0}$. The starting point is Eq. (10); substitution therein of the third of Eqs. (A-1) and expansion about $\eta_{r}=\eta_{r 0}$ lead to

$$
\hat{H}^{\prime}{ }_{2}\left(\eta=\eta_{r 0}{ }^{+} ; \eta_{r 0}\right)-\sigma f_{0 r}=\hat{H}^{\prime}{ }_{2}\left(\eta=\eta_{r 0}{ }^{-} ; \eta_{r 0}\right)+\hat{c}^{\prime}\left(\eta=\eta_{r 0}+\eta_{r 0}\right)
$$

We next examine whether $\hat{H}_{2}$ is continuous at $\eta=\eta_{r 0}$; if the second of Eqs. (A-4) is integrated across $\eta_{r 0}$ and if Eq. (A-7) is introduced, there results

$$
\hat{H}_{2}\left(\eta=\eta_{r 0}{ }^{+} ; \eta_{r 0}\right)=\hat{H}_{2}\left(\eta=\eta_{r 0}{ }^{-} ; \eta_{r 0}\right)-1
$$

which indicates that both $\hat{H}_{2}$ and $\hat{H}_{2}^{\prime}$ are discontinuous at $\eta_{r 0}$.

The desired derivative with respect to $\eta_{r}$ can be calculated from the solution on either side of $\eta_{r 0}$, for example, as

$$
-\frac{\partial H_{\psi 1 r}}{\partial \eta_{r}}=H_{\psi 1}^{\prime}\left(\eta=\eta_{r 0}{ }^{+} ; \eta_{r 0}\right)+c^{\prime}{ }_{0 r} \hat{H}_{2}\left(\eta=\eta_{r 0}{ }^{+} ; \eta_{r 0}\right)
$$

Thus we see that the derivatives we seek are given by quantities already known from the solutions of Eqs. (7)-(9) and by the new quantities $\hat{c}^{\prime}\left(\eta=\eta_{r 0}{ }^{+} ; \eta_{r 0}\right), \hat{H}_{1}\left(\eta=\eta_{r 0} ; \eta_{r 0}\right)$ and $\hat{H}_{2}\left(\eta=\eta_{r 0}{ }^{+} ; \eta_{r 0}\right)$ determined numerically.

\section{NOMENCLATURE}

$\begin{array}{ll}A_{f}, B_{f} & \text { integration constants in asymptotic solution } \\ a & \text { rate of strain parameter } \\ B & \text { preexponential factor } \\ C & \text { progress variable } \\ D & \text { Damköhler number } \\ f & \text { modified stream function } \\ H & \text { nondimensional enthalpy parameter, } H=(h-1) / \tau \\ h & \text { nondimensional enthalpy } \\ L e & \text { Lewis number } \\ m & \text { parameter relating to the rate of creation of product } \\ T & \text { nondimensional temperature } \\ u & \text { radial velocity component } \\ w & \text { volumetric rate of product creation }\end{array}$




\section{Greek}
$\Delta, \Delta_{a}, \Delta_{n}, \delta$ reduced Damköhler parameters
$\varepsilon, \varepsilon_{n}, \varepsilon_{\infty}$
expansion parameters
$p$
nondimensional mass density
$\eta$
$a, \beta, \gamma$
similarity variable

$\tau$
$\kappa, \kappa_{f}$
$\sigma$
$\nu$
$\xi, \zeta$
$\chi$
$\chi_{L}, \chi_{L n}$
$\lambda$
parameters in inner solutions for nonadiabatic flamelets heat release parameter parameter arising in asymptotic $(\eta \rightarrow \pm \infty)$ solutions Prandtl number kinematic viscosity stretch coordinates within reaction zones parameter arising in nearly adiabatic analysis parameters arising in the analysis of near unity Lewis numbers parameter arising in analysis of highly strained flamelets

$\begin{array}{ll}\text { Subscripts } & \\ a & \text { activation } \\ o & \text { reactant stream } \\ r & \text { reaction zone } \\ \infty & \text { product stream } \\ 0 r & \text { zero order at reaction zone } \\ 1 r & \text { first order at reaction zone } \\ e & \text { extinction or extrema } \\ H 1 & \text { first order enthalpy perturbation } \\ \psi 1 & \text { first order Lewis number perturbation } \\ 01 & \text { first order Lewis number perturbation }\end{array}$

